Erschienen in: Harras, Gisela (Hrsg.): Die Ordnung der Wörter. Kognitive und lexikalische Strukturen. - Berlin, New York: de Gruyter, 1995. S. 138-178. (Institut für deutsche Sprache. Jahrbuch 1993)

\title{
FRITZ HERMANNS
}

\section{Kognition, Emotion, Intention}

\section{Dimensionen lexikalischer Semantik}

\section{Vorbemerkung ${ }^{1}$}

Ich bin zu dieser Tagung eingeladen worden und zu diesem Referat, damit ich ein Kontrastprogramm bestreite. Diese Tagung sucht ja sonst das Wesen lexikalischer Semantik im Bereich des Kognitiven. Das sagt jedenfalls ihr Titel, demzufolge die Ordnung der Wörter wesentlich in einem irgendwie gearteten Zusammenhang von kognitiven und lexikalischen Strukturen aufzufinden ist. In einem Vortrag aber sollte auch das Andere der lexikalischen Semantik abgehandelt werden, also neben $\mathrm{Kog}$ nition auch Emotion und Intention; mit anderen Worten: neben Denken und Erkennen auch das Fühlen und das Wollen, wie es sich in Sprache und in Lexik ausdrückt. Ich bezeichne Kognition und Emotion und Intention als Dimensionen lexikalischer Semantik. Damit möchte ich betonen, $\mathrm{daB}$ es in der lexikalischen Semantik nicht allein auf Kognitionen, sondern ebenso auf Emotionen und auf Intentionen ankommt, weil sie, wie ich gern plausibel machen möchte, in der Lexik ebenso versprachlicht sind wie Kognitionen. Und zwar nicht nur sozusagen nebenbei, wie das der Terminus Konnotation besagt. ${ }^{2}$ Manchmal werde ich die Dimensionen lexikalischer Semantik kürzer auch die Zeichendimensionen nennen. ${ }^{3}$

Meine Trias von Begriffen wird Sie wohl ein wenig irritieren, weil es ungewöhnlich ist, daB man, in einem Atemzuge sozusagen, Kognition und Emotion und Intention zusammenfaAt. Ich werde das erklären. Vorerst möchte ich Sie darum bitten, diese Trias einfach einmal hinzunehmen. Und sich einzulassen auf den folgenden Gedankengang, bei dem es also um die Relevanz von Emotionen und von Intentionen für die lexikalische Semantik gehen soll. Um die, wie ich sagen möchte, lexikalisierten Emotionen und die lexikalisierten Intentionen. Denn daß Kognitionen le-

1 An der Form des Vortrags habe ich in dieser redigierten Fassung meines Beitrags nur Retuschen angebracht und sie im wesentlichen beibehalten. Zur Vermeidung von Irritationen weise ich hier noch auf Folgendes hin:

a) Kursivschreibung benutze ich oft auch da, wo ich ein Wort verwende (also nicht nur da, wo ich zitiere), nämlich dann, wenn ich die Aufmerksamkeit bei der Lektūre auf das Wort (statt nur auf die gemeinte Sache) lenken möchte, außerdem benutze ich sie zur Betonung; b) alle übrigen (außer dieser ersten) Anmerkungen stehen wegen ihrer z.T. exorbitanten Länge am Ende dieses Beitrags. 
xikalisiert sein können, das ist linguistischer Konsensus, und ich brauche das nicht eigens auszuführen.

Ich beginne damit, daß ich erstens etwas sage zum Zusammenhang von Kognition und Emotion und Intention in $\ddot{A} u ß e r u n g e n$, also vorab etwas sage zum Zusammenhang der Zeichendimensionen lediglich in der parole, und nicht schon in der langue. Zweitens rede ich vom emotiven Anteil in der lexikalischen Bedeutung eines Wortes, also von den lexikalisierten Emotionen. Überleitend führe ich dann drittens etwas aus zur Frage des Zusammenhangs von Intention und Präskription, d.h. zur Frage des semantischen Zusammenhangs von Wollen einerseits und Sollen andererseits, die nämlich evidentermaßen so zusammenhängen, daß ein ausgesagtes Sollen übereinstimmt mit dem Wollen dessen, der es aussagt; so daB ausgesagte Präskriptionen in direkter Weise Intentionen (eines Sprechers in Bezug auf Tun und Lassen seines Adressaten) zeigen. Viertens geht es mir dann um den präskriptiven Anteil in der lexikalischen Bedeutung eines Wortes, also um die lexikalisierten Präskriptionen, die jedoch nach dem zuvor Gesagten gar nichts anderes sind als lexikalisierte Intentionen. Füftens und abschließend deute ich im Wege eines Ausblicks an, worauf sich der Zusammenhang von Kognitionen, Emotionen, Intentionen gründen könnte.

\section{Kognition und Emotion und Intention in Äußerungen}

Zum Zusammenhang von Kognition und Emotion und Intention in Äußerungen: um ihn darzulegen, möchte ich das Bühlersche Szenario verbaler Kommunikation benutzen, also Bühlers Organon-Modell der Sprachverwendung (Bühler 1934, S. 28). Ich rufe dazu Bühlers Schema in Erinnerung, wobei ich aber einige Akzente anders setze, als das Bühler selbst tut. Bühlers Schema hat, Sie wissen es, auf seiner linken Seite eine Position für einen Sprecher, Bühler nennt ihn Sender, auf der rechten Seite eine Position für einen Hörer, Bühler sagt Empfänger; oben oder hinten hat es eine Position für das, worüber in der Kommunikation gesprochen wird, die Gegenstände oder Sachverhalte. In der Mitte schließlich ist ein Kreis mit einem Dreieck, dessen Seiten den genannten Positionen zugekehrt sind. 


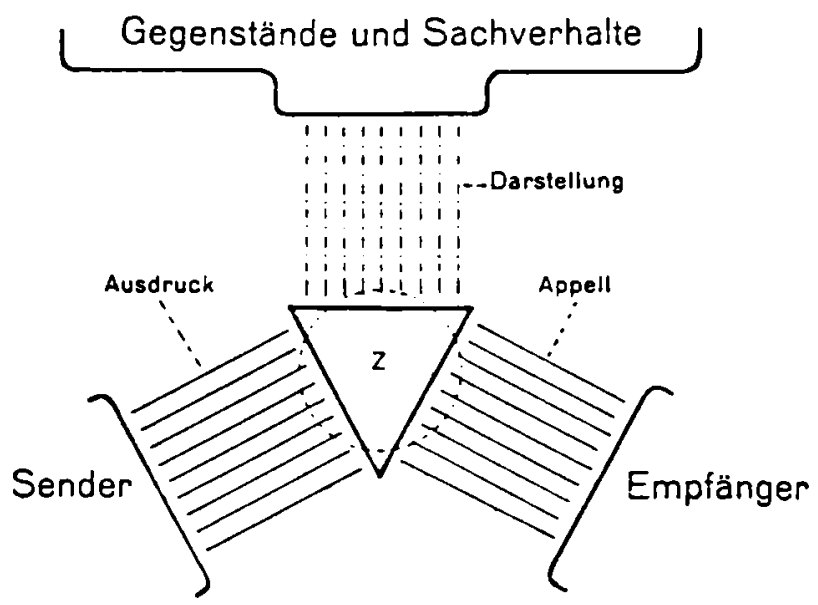

Dieser Kreis mit Dreieck steht nun für die Zeigehandlung, wie ich sie hier nennen möchte, Bühler nennt sie Zeichen. Der Kreis symbolisiert das je konkrete Schallereignis (token), aber wie es wahrgenommen wird als einem Typ von Zeigehandlung zugehörig, dafür steht das Dreieck. Kreis und Dreieck überschneiden sich, und das versinnbildlicht, daß bei der Perzeption der Zeigehandlung einerseits das Wahrgenomme in seiner Vielfalt auf das für den Typ der Zeigehandlung Relevante reduziert wird (Bühler spricht von dem Prinzip der abstraktiven Relevanz), andererseits jedoch nach Maßgabe des Typs der Zeigehandlung umgekehrt auch komplettiert wird (Bühler spricht von einer apperzeptiven Ergänzung). Eine Äußerung ist, so betrachtet, die vokale und verbale Zeigehandlung, die ein Sprecher ausführt und ein Hörer wahrnimmt (aber auch der Sprecher selber wahrnimmt), wie er sie im Wege der Gestaltbereinigung (d.h. der Reduktion auf die Gestalt) und der Gestaltergänzung (Ergänzung zur Gestalt) einem Typ der Zeigehandlung zugeordnet hat, d.h. erkannt hat. ${ }^{4}$

Ich lasse jetzt den Sprecher eine solche Zeigehandlung machen. Um ein allgemein bekanntes Beispiel vorzuführen, lasse ich den Sprecher rufen: Hilfe! Hilfe!

Bühlers Paradox: ein Zeichen ist drei Zeichen

Inwiefern ist dieser Hilferuf ein Zeichen? Das ist in der Tat die Frage Bühlers. Nun, ein Zeichen ist ein Zeichen, insofern es etwas - das $G e-$ 
zeigte - zeigt, das wußte man schon immer. Und zwar etwas anderes zeigt als nur sich selber. Insofern man daraus schließen kann auf das Gezeigte (wenn auch nicht im Wege eines deduktiven Schlusses, wie ich vorsichtshalber hier betone), so sagen es Prieto $(1975$, S. 18) ebenso wie Keller $(1992$, S. 327). Was bei Bühler nun epochemachend neu ist gegenüber einer Tradition der Semiotik seit der griechischen Antike ist, Sie wissen es, daB Bühler sagt: Die Äußerung ist nicht nur einfach Zeichen, sondern dreifach.

Denn sie zeigt als Zeigehandlung uno actu dreierlei Verschiedenes. Gegenstände oder Sachverhalte stellt sie dar, wie Bühler sagt. Selbstverständlich nicht die Gegenstände oder Sachverhalte selber, wie sie etwa "objektiv” bzw. „wirklich” wären, sondern so, wie sie der Sprecher denkt bzw. wahrnimmt, also wie sie sind in dessen Kognition. Und auch diese Kognition zeigt sie natürlich nur, soweit der Sprecher sie versprachlicht. Hier in unserem Beispiel ist das Dargestellte etwa: Hier ist jemand in Gefahr. Ich brauche dringend Beistand. Es ist also nicht so, daß der Hilferuf darstellungsfunktional als solcher leer ist; vielmehr sagt er das Bestehen eines Sachverhaltes (nämlich einer Notlage) mit aus, wenn er ihn auch nicht (im Sinne einer Assertion) behauptet. ${ }^{5}$ Darin, daß es Gegenstände oder Sachverhalte darstellt, besteht die Darstellungsfunktion bzw. die Symbolfunktion, wie Bühler sagt, des Zeichens. Vermöge derer ganz allein es schon ein Zeichen wäre.

In Bezug auf ihren Aktor ist die Zeigehandlung Ausdruck oder auch Symptom, insofern hat sie eine Ausdrucks- oder auch Symptomfunktion, so nennt es Bühler. Was sie bezüglich ihres Aktors zeigt, das kann so manches sein, doch insbesondere zeigt sie auch die Emotion des Aktors. ${ }^{6}$ In unserem Beispiel ist die Emotion die Angst, die zu dem Hilferuf dazugehört. Denn wenn er keine Angst zum Ausdruck bringt, dann ist der Hilferuf als Hilferuf mißlungen. Und vermöge dessen, daß die Zeigehandlung Ausdruck ist von etwas, ist sie abermals ein Zeichen.

In Bezug auf ihren Adressaten schließlich zeigt die Zeigehandlung, was der Sprecher möchte, daß der Hörer tun soll. Bühler spricht von der $A p$ pellfunktion bzw. der Signalfunktion des Zeichens, die in unserem Beispiel sozusagen explizit ist. Hilfe! Hilfe! heißt: Wer immer du auch bist, der diese Botschaft hört - laß bitte alles stehen und liegen, was du jetzt gerade tust, und bringe augenblicklich Hilfe! Oder wenn du selber keine Hilfe bringen kannst, dann hole Hilfe! Also eine Äußerung läßt nicht nur eine Kognition erkennen und bringt ferner nicht nur eine Emotion zum Ausdruck, sondern zeigt dem Hörer auch die Intention des Sprechers in Bezug darauf, wie er, der Hörer, auf die Äußerung als Zeigehandlung reagieren soll. Vermöge dessen, daß die Zeigehandlung auch die intendierte 
Reaktion des Hörers auf die Zeigehandlung anzeigt, ist sie demnach noch auf eine dritte Weise Zeichen.

Dreifach also sind nach Bühler die Funktionen der verbalen Zeigehandlung, wie er sie an seinem Organon-Modell entwickelt. Drei verschiedene Funktionen hat sie für den Sprecher, der sie wie ein multifunktionales Werkzeug (organon) benutzt, das drei verschiedene Funktionen hat, die aber hier bei jeder einzelnen Verwendung simultan zum Zuge kommen, wenn er uno actu etwas darstellt, ausdrückt und signalisiert. Dementsprechend hat sie gleichfalls drei verschiedene Funktionen für den Hörer. Er erkennt anhand der Zeigehandlung simultan ein Dargestelltes (Kognition des Sprechers) und ein Ausgedrücktes (Emotion des Sprechers) und auch ein Gewolltes (Intention des Sprechers). Und nur, wenn er alles dies erkennt, hat er verstanden, was der Sprecher meinte.

\section{Verstehen schließt die Emotionen und die Intentionen mit ein}

Das Verstehen eines Zeichens, also einer Zeigehandlung, ist in seinem Kern nichts anderes als das Erkennen dieser Zeigehandlung. Im Gelingensfalle: so, wie sie gemeint ist. Dazu nun gehört bei einer Zeigehandlung immer zweierlei Erkennen. Beiderlei Erkennen einer Zeigehandlung nennen wir Verstehen. ${ }^{7}$ Erstens das Erkennen der gemeinten, der gewollten Zeigehandlung selber, der Gestalt der Zeigehandlung oder, wie man linguistisch gerne sagt, der Form des Zeichens, die man dann containermetaphorisch unterscheidet von dem sogenannten Inhalt. Erstens also geht es beim Verstehen darum, das Bezeichnende als solches zu erkennen, das signifiant. Wenn dies nicht gelingt, dann sagen wir vielleicht: Ich habe das akustisch nicht verstanden.

Zweitens und vor allem geht es beim Verstehen auch um das Erkennen des gemeinten signifié; wie schon gesagt, im Wege eines Schlusses. Vom signifiant wird auf das signifié geschlossen. Dabei schließt man also aus der Form des Zeichens, aus der Art und Weise des vokalen Zeigehandelns, auf das, was der Aktor damit zeigen wollte. Wenn dies nicht gelingt, dann sagen wir: Ich habe nicht verstanden, was du meinst.

Signifié, das wird gewöhnlich (aber nicht gerade glücklich) übersetzt mit das Bezeichnete. Ich übersetze einmal: das Gezeigte. ${ }^{8}$ Das Gezeigte ist nichts anderes als das Gemeinte, also die Bedeutung einer Zeigehandlung (Bedeutung hier im Sinne des Bedeuteten der Zeigehandlung). Das Gezeigte ist nichts anderes als die Gesamtbedeutung einer Zeigehandlung, sollte man vielleicht verdeutlichend genauer sagen. Denn nicht selten wird $\mathrm{ja}$ in der Linguistik (so von Austin (1962) und von Bierwisch (1980)) die Bedeutung von Bedeutung (oder meaning) reserviert für das, 
was ich den deskriptiven (oder kognitiven) Anteil der Gesamtbedeutung nenne, den man so vom expressiven und vom präskriptiven Anteil der Gesamtbedeutung der verbalen Zeigehandlung unterscheidet; letzterer heißt dann die illokutionäre Rolle oder auch der kommunikative Sinn der Äußerung, der Zeigehandlung. Bühlers einprägsames Schema führt vor Augen, da $\beta$ in der Gesamtbedeutung der verbalen Zeigehandlung mehr gezeigt ist als bloß die Bedeutung sensu stricto und daB also das Verstandenhaben einer Äußerung und eines Satzes mehr ist als bloß „wissen, was der Fall ist, wenn er wahr ist". ${ }^{9}$

\section{Drei grammatische Personen, also auch drei Sprachfunktionen?}

Seine hohe Plausibilität verdankt das Bühlersche Modell vor allem seiner Übersichtlichkeit, es macht die Dinge einfach; allzu einfach, wie ich meine. Ganz heterogene Fragen und Gedanken bringt es in ein scheinbar homogenes Schema. Bühlers Dreierschema nämlich faßt, doch ohne $\mathrm{daB}$ dies diskutiert und explizit gemacht wird, höchst Verschiedenes in eins zusammen: a) eine Theorie der für die Kommunikation entscheidenden Faktoren, d.h. ein Kommunikationsmodell; b) eine Theorie der Zeichensorten; c) eine Theorie der Zeichenrelationen; und d) eine Theorie der Sprachfunktionen. Diese ganz verschiedenen Theorien bringt das Bühlersche Modell zur Deckung. Das gelingt nicht ohne ein gewisses Maß begrifflicher und theoretischer Gewaltsamkeit.

Letztlich gibt es hier genau drei - nicht weniger, nicht mehr - Sprachfunktionen, weil es drei grammatische Personen gibt. Bühlers Kommunikationsmodell reproduziert die Trias der grammatischen Personen (ich, $d u, e r / s i e / e s$ ), denen nämlich seine Positionen (die des Senders, des Empfängers und der Gegenstände/Sachverhalte, neben der des Zeichens selber) Punkt für Punkt entsprechen. Diese Positionen sind zugleich die streng getrennten Referenzbereiche für die Zeichensorten des Symbols, Symptoms, Signals. Diese wiederum sind aber auch noch dadurch unterschieden, daß sie ihre Zeichenhaftigkeit verschiedenen Zeichenrelationen (Art der Relation von Zeichen und Bezeichnetem) verdanken. Und es haben diese Zeichensorten auch noch ihre jeweils eigenen, besonderen Funktionen. ${ }^{10}$

So entsteht ein Bild der in der jeweils einen Zeigehandlung kopräsenten drei Funktionen (respektive Zeichensorten), wonach diese drei Funktionen strikt getrennt sind. Bühler hebt die Eigenständigkeit der Zeichendimensionen und der Zeichensorten so hervor, daß man den Eindruck hat, es hätten eigentlich die Dimensionen miteinander nichts zu tun; als wäre es geradezu ein Wunder, daß die Zeichensorten des Symbols, Symptoms, Signals in der verbalen Zeigehandlung in concreto eins sind. 
So fremd sind sie einander. Die Gesamtbedeutung ist bei Bühler bloß das Resultat der Addition der einzelnen Bedeutungen in jeder der drei Zeichendimensionen.

\section{Zwar drei Zeichen, aber trotzdem eine Einheit}

Dabei wissen wir doch aus Erfahrung und aus Reflexion: die einzelnen Bedeutungen sind nicht unabhängig voneinander. Denn in aller Regel ist es so, daß eine ausgedrückte Emotion mit einer dargestellten Kognition und einer offenbarten Intention zusammenpaßt, so daß man von dem einen auf das andere sogar schließen kann. Im Verstehen der Gesamtbedeutung einer Äußerung sind daher auch die Komponenten des Gesamtverstehens nicht bloß additiv vereinigt. So paßt auch in unserem Beispiel eines Hilferufs die damit dargestellte Notsituation zur darin ausgedrückten Angst, und dieses beides paßt dazu, daß dieser Hilferuf als ein Appell signalisiert, der Hörer möge Hilfe leisten oder holen. Darstellung, Ausdruck und Appell sind sozusagen solidarisch. Ob eine Äußerung perlokutionär erfolgreich ist, hängt wesentlich auch von der ausgedrückten Emotion und von der dargestellten Kognition des Sprechers ab, die in der Äußerung als Zeigehandlung konsubstantial sind mit der offenbarten Intention. Dergestalt, daß diese drei ein Ganzes, eine Einheit bilden, das als Ganzes und als Einheit wahrgenommen werden soll und wird.

Wohlgemerkt, ich spreche hier, in diesem ganzen ersten Abschnitt meines Vortrags, von der Äußerung, von der parole. Gibt es, frage ich nun weiter, etwas Ähnliches auch im Bereich der langue und im Bereich der Lexik? Gibt es Wörter, die zugleich - in Personalunion gewissermaßen der Darstellung im Sinne Bühlers dienen und dem Ausdruck einer Emotion? Oder auch zugleich der Darstellung und dem Appell? Also frage ich jetzt weiter nach den lexikalisierten Emotionen und dann nach den lexikalisierten Intentionen.

\section{Lexikalisierte Emotionen}

Was für Wörter dienen überhaupt dem Ausdruck von Gefühlen? Ohne Zögern wird man vielleicht sagen: die Gefühlswörter, wie man sie genannt hat. Das sind Wörter wie z.B. Liebe, Haß und Eifersucht und Angst und Wut und Trauer, nebst den Verben und den Adjektiven, die dazugehören. ${ }^{11}$

\section{Quasi-psychologische Vokabeln}

Solche Wörter möchte ich für meine Zwecke hier als quasi-psychologische bezeichnen. Denn sie dienen zur Benennung von Gefühlen und Gemüts- 
zuständen insbesondere in deskriptiver Absicht. In der Regel sind sie selber aber gar nicht emotiv und expressiv. Peter ist auf Dieter eifersüchtig, dieser Satz bezeichnet eine Emotion, doch bringt er selber keine Emotion zum Ausdruck. Hier wird durch Gebrauch des Wortes eifersüchtig eine Emotion benannt und zugeschrieben, aber das geschieht auf gänzlich kühle Art und Weise, sozusagen diagnostisch. Peter ist verliebt, das unterscheidet sich, was seine Expressivität betrifft, wohl kaum von $P e$ ter ist erkältet. Es ist gleichfalls eine Diagnose. Darum also nenne ich dergleichen Wörter quasi-psychologisch.

Allerdings, die Wörter dieses Typus können auch verwendet werden, um Gefühle auszudrücken. Wenn man beispielsweise mit Emphase sagt: Ich hasse das! oder auch: Das macht mich wütend! oder auch: Ich habe Angst!, dann ist das nicht allein die Deskription von einer Emotion, es ist zugleich auch deren Ausdruck. Kürzlich habe ich eine alte Dame sagen hören, von dem Tag, an dem ihr Mann beerdigt wurde: Vor dem Tag hab' ich mich so gefürchtet. Das war alles andere als emotionslos. Trotzdem hat ein solcher Satz noch etwas Distanziertes. Denn er ist der Form nach eine Selbstbeschreibung und nicht eigentlich der Ausdruck des Gefühls.

Alle diese Sätze wie Ich habe Angst, Ich freue mich, Ich liebe dich sind Sätze in der Ersten Singularis mit dem Personalpronomen ich, und dieses Wörtchen ich bedeutet, daB im Bühlerschen Modell die Positionen für den Sprecher und die Gegenstände/Sachverhalle gleich besetzt sind; daß der Sprecher selber Gegenstand der eigenen Rede ist, so wie beim $d u$ der Hörer. Das bedeutet aber eben, daß die Sätze wie Ich liebe dich der Form nach deskriptiv sind und sich darin gar nicht unterscheiden von den Sätzen in der dritten Singularis wie z.B. Sie liebt ihn. Ich finde auch, der Satz Ich liebe dich drückt das Gefühl der Liebe in der Regel gar nicht aus, er hat stattdessen etwas Förmliches und Feierliches, wie es dem Ernst der Lage angemessen ist, wenn jemand einen solchen folgenschweren Ausspruch tut. Jedenfalls, ich resümiere, scheint mir, daß die Wörter des Gefühlswortschatzes allenfalls nur sekundär dem Ausdruck von Gefühlen dienen - Ausdruck nach wie vor im Sinne Bühlers - und in erster Linie deren distanzierter, deskriptiver, quasi-psychologischer Benennung. ${ }^{12}$

\section{Empfindungswörter}

Gibt es Wörter, wo das anders ist und die primär dem Ausdruck von Gefühlen und Affekten dienen? Solche Wörter sind bestimmt die Schimpf- und Kosenamen. Darauf komme ich noch kurz zu sprechen. 
Ferner gibt es die Partikeln wie das international berühmte deutsche Wörtchen ach und auch z.B. pfui und au. In der exzellenten, wie ich finde, Neubearbeitung des Paulschen Wörterbuchs heißen sie Empfindungswörter. Burkhardt, der sie dort beschreibt, hat beispielsweise $p f u i$ charakterisiert als: „1. Ausdruck von Ekel u. Widerwillen” (mit dem Beispiel - Abraham a Sancta Clara über einen Leichnam - „Pfuy! er stinkt schon”). „2. (emotionaler) Ausdruck von Ablehnung, Abscheu und Verachtung”. Dazu heißt es weiter: „nicht selten mit dem Vorwurf sittlichmoralischen Fehlverhaltens", und es folgt das schöne Beispiel aus dem Struwwelpeter:

Sieh einmal, hier steht er, $p$ fui, der Struwwelpeter!

An den Händen beiden ließ er sich nicht schneiden seine Nägel fast ein Jahr; kämmen ließ er nicht sein Haar.

Pfui, ruft da ein jeder:

Garstger Struwwelpeter! ${ }^{13}$

Darauf folgt ein zweiter Hinweis zum Gebrauch des Wortes: „nicht selten mit der Aufforderung, sich zu schämen", was die etwas Älteren von uns noch aus der Sprache der Erziehung kennen: Pfui, schäm' dich, stell dich in die Ecke! hieß es früher. Aufschlußreich auch für die Synchronie ist schließlich noch die diachrone Auskunft: „wohl urspr. lautl. Nachbildung des Geräusches beim Ausspeien".

Ich zitiere den Artikel deshalb so ausführlich, weil er so verfaßt ist, daß man denken könnte, der Verfasser hätte meinen Vortrag schon gekannt und hätte mir mit dem Artikel Unterstützung geben wollen. In den anderen Artikeln dieses Wörterbuches nämlich findet man in spitzen Klammern die Bedeutungen der Lemmawörter angegeben. Das ist hier nicht der Fall. Sondern es heißt hier an der Stelle, wo sonst die Bedeutungsparaphrase anzutreffen ist, und ohne spitze Klammern: Ausdruck von Ekel oder Abscheu. Das besagt: Das Wörtchen pfui bezeichnet nicht den Ekel, wie es die Vokabel Ekel tut, als psychologische Vokabel. Sondern pfui ist, wie es hier ja wörtlich heißt, der Ausdruck eines Ekels oder Abscheus, also sozusagen selbst der ausgedrückte Ekel oder Abscheu, der in der verbalen Zeigegeste sich gewissermaßen ausagiert. Wie er das auch durch reales Spucken tun kann.

Deshalb ist bei diesem wie bei anderen solchen Wörtern auch die nonverbale Geste, die damit einhergeht, nichts dem Wort nur Äußerliches, das dem Wort zum besseren Verständnis des Gemeinten redundanter- 
weise noch hinzugefügt wird. Sondern eher umgekehrt: Das Wort wird zur Verdeutlichung der körperlichen Geste ausgesprochen. Im Fall des pfui spannt sich dabei der ganze Körper an und zieht sich das Gesicht zusammen; der Blick wird starr, die Lippen werden schmal, der Mund wird klein, der Kopf macht einen kleinen Ruck nach hinten, um dann wieder vorzuschnellen. Die fest gepreBten Lippen öffnen sich zu einem Spalt. Und bloß, was da hervorgestoßen wird aus diesen festgepreßten Lippen, ist nicht wirklich Speichel, sondern dessen Surrogat: das Wörtchen $p f u i$, das scharf und häßlich zischend ausgesprochen wird. Das also nur ein Teil der Abscheugeste ist. ${ }^{14}$

Entsprechend gilt auch für die anderen Empfindungswörter, daß im typischen Gebrauch sie immer Teil sind einer körperlichen Geste, daB sie also non-verbal und para-sprachlich eingebunden sind in eine ganzheitliche Zeigehandlung. ${ }^{15}$ Beispielsweise, um ein angenehmeres Empfindungswort noch anzuführen, das bereits erwähnte ach, bei dem man vorher Luft holt, denn man atmet dann das Wort, wenn man es ausspricht, sozusagen aus mit einem tiefen Seufzer: ach! Das Wort ist hier gewissermaßen selbst der Seufzer. Dabei entspannt man sich nach allen Regeln der Entspannungskunst, die Züge werden weich, die Muskeln locker. Also man kann nur jedem raten, öfter einmal ach! zu sagen.

\section{Affektive Adjektive}

Gibt es außer den genannten auch noch andere Wörtertypen, die speziell dem Ausdruck von Gefühlen dienen? Eine Gruppe solcher Wörter interessiert mich insbesondere. Ich erlaube mir, aus einem sicherlich nicht allgemein bekannten Aufsatz (Hermanns 1986) zu zitieren, weil ich möchte, daß Sie nacherleben können, wie ich auf die Gruppe dieser Wörter aufmerksam geworden bin. Ich habe dort berichtet, wie ich zum Bewunderer und Freund des damals neuen Duden Universalwörterbuchs (im folgenden kurz: $D U W$ ) geworden bin.

Nämlich dadurch, daß ich dort die Wörter niedlich, lieb und goldig nachgeschlagen habe. Da stand unter niedlich folgende Bedeutungsparaphrase: „durch seine hübsche Kleinheit, Zierlichkeit, durch zierliche, anmutige Bewegungen o.ä." - und jetzt kommt das Entscheidende "Gefallen erregend, Entzücken hervorrufend"; hinzugefügt waren noch die Synonyme lieb, goldig, reizend. Und zum Lemma goldig konnte ich dort lesen: „(ugs.) in seiner äußeren Erscheinung in einer Weise reizend, daß man es" - und jetzt kommt wieder das Entscheidende - „mit Rührung u. Zärtlichkeit feststellt”. 
Das ist, wie ich finde, schön gesagt, vor allem aber ist es treffend. Niedlich, lieb und goldig sind, wenn ich es richtig sehe, regionale Varianten einer und derselben sprachlichen Gebärde. Hier in Mannheim sagt man goldig (beispielsweise, wie mir mein Gewährsmann Uwe Zipf berichtet: Des is e goldisch Knuddel!), und in Hamburg sagt man niedlich oder süß (z.B. in dem Ausruf: Nein, wie ist sie süß), in Wien dagegen sagt man lieb und herzig. Meistens sagt man es von Babys oder kleinen Kindern.

Was man damit deskriptiv von Babys oder kleinen Kindern sagt, ist allerdings nicht viel. Etwas Relevantes sagt man damit aber über sich aus. Das ist goldig! - das bedeutet in der Tat, wie es das Wörterbuch uns sagt: Ich bin entzückt, ich bin gerührt, mein Herz ist voller Zärtlichkeit, bei diesem Anblick. Und offensichtlich drücken diese Wörter solche Emotionen aus, und sie benennen sie nicht diagnostisch.

Auf alle Fälle, wenn sie so gesprochen werden, wie es ihnen angemessen ist, in Tonfall, Timbre, Rhythmus, Mimik, Gestik integriert in eine ganzheitliche emotive Zeigehandlung, die als Ganze auf den Hörer und Betrachter wirken soll und wirkt. Das ist bei allen solchen Wörtern ganz genauso wie bei den Empfindungswörtern, die wir gerade hatten. Um den Preis terminologischer Verwirrung kann man sagen, daß es sich hier gleichfalls um Empfindungswörter handelt.

Was ist das Besondere an der semantischen Struktur von solchen Wörtern? Nun, sie passen nicht ins Bühler-Schema. Nicht problemlos. Scheinbar über gar nichts anderes als Gegenstände/Sachverhalte redend, gebe ich bei der Verwendung solcher Wörter gleichermaßen etwas über mich, den Sprecher, zu verstehen. Denn der Sprecher scheint sich auf den Gegenstand zu konzentrieren, wenn er ausruft: Das ist aber niedlich! Und der Form nach ist der Satz auch in der Tat desselben Typs wie etwa Das ist rot und Das ist rund, wo wirklich über die Beschaffenheit von Gegenständen etwas ausgesagt wird. Wenn ich aber sage: Das ist niedlich! - rede ich als Sprecher ebenso auch von mir selber. So daß dieser Satz im Bühler-Schema quer steht, nämlich gleichermaßen auf die Gegenstände/Sachverhalte und den Sprecher sich beziehend.

Wollte man das prädikatenlogisch formulieren, müßte man wohl sagen: goldig, lieb und niedlich sind entgegen erstem Anschein nicht einstellige, sondern zweistellige Prädikatoren oder Prädikate, und man müBte also dafür schreiben nicht $P(x)$, sondern $Q(x, y)$, wobei $P$ die Variable für ein affektives Adjektiv wie niedlich wäre, $Q$ die Variable für ein Emotionswort, $x$ für das Subjekt des Satzes, $y$ ein Name für den Sprecher. ${ }^{16}$ Zweistellige Prädikate, d.h. Relationen. Das Verwendungsschema solcher Adjektive wäre demnach, daß man, wenn man etwa sagt: $x$ ist $P$, damit 
sagt: $x$ ist so geartet, daß ich, wenn ich es betrachte oder daran denke, die-und-die Gefühle habe. Oder, um es anders auszudrücken: $x$ bewirkt, daß ich die-und-die Gefühle habe. Das heißt, daß die Formel $P(x)$ in solchen Fällen explizit zu machen wäre durch die Formel $C A U S(x, Q(y))$, wobei $Q(y)$ bedeuten würde, daß $y$ die Emotion $Q$ "hat”. Danach wären solche Adjektive also zu beschreiben als kausative affektive Adjektive.

\section{Kausative Adjektive}

In der Tat besteht hier eine Ähnlichkeit mit kausativen Verben. ${ }^{17}$ Wir erkennen diese Ähnlichkeit am besten, wenn wir an die deverbalen Adjektive denken - oder an die Partizipien - mit derselben logischen Struktur wie niedlich.

Beispielsweise reizend (wenn es auch semantisch mit dem Verbum reizen heute nicht mehr viel zu tun hat). Dieses ganz besonders in dem Ausruf Das ist aber reizend! heute oft als affektiert empfundene, weil bildungs- oder auch kleinbürgerliche Synonym von niedlich und von goldig ist semantisch-logisch gleichfalls so zu deuten, daß man damit aussagt, daß etwas bewirkt, daß man beglückt ist. Das Kausative daran ist hier sozusagen angedeutet in der Endung -end.

Deutlicher noch ist der Zusammenhang bei echten Partizipien und bei Adjektiven, die man, wenn man will, als Partizipien verstehen kann, z.B. bei entzückend; denn entzückend ist, was mich entzückt. Oder auch bei ermüdend. Das ermüdende Gespräch ist eines, das bewirkt, daß ich von einem wachen Zustand in den der Ermüdung übergehe. Eine nervtötende Fernsehsendung ist eine solche, die bewirkt, daß mir der letzte Nerv getötet wird. Eine atemraubende Aktion ist so beschaffen, daB sie bewirkt, daß mir der Atem stillsteht, usw. Aufregend ist in unserem Zusammenhang ein gutes Beispiel, weil es uns zu einer unbezweifelbaren Emotion zurückführt. Etwas ist aufregend dann und in dem $\mathrm{MaB}$, wie es bewirkt, $\mathrm{da} B$ ich in den Zustand einer Aufgeregtheit übergehe. Ganz genauso sind semantisch-morphologisch rührend und empörend und erheiternd zu analysieren.

In allen diesen Fällen, also bei den Partizipien - oder partizipialen Adjektiven, wie man sie vielleicht vorsichtig nennen sollte - ist das Kausative oder Faktitive der Bedeutung sozusagen explizit im kausativen Verbstamm und der Endung. Das sind die - wenn man so will - trivialen Fälle. Nichts hindert uns jedoch, die Analyse, die in diesem expliziten Fall sich aufdrängt, auch auf solche Adjektive anzuwenden, wo das Kausative nicht durch eigene Morpheme angezeigt wird, wie bei niedlich oder goldig oder herzig. Eine Zwischenstellung zwischen Ex- und Im- 
plizitheit nehmen solche Adjektive ein, bei denen ihre Etymologie noch durchscheint (synchronisch ausgedrückt: die semi-motiviert bzw. semitransparent sind), wie erfreulich und entsetzlich und abscheulich (oder auch z.B. ekelhaft und eklig), wo die Endung -lich (bzw. -haft und -ig) dieselbe Rolle spielt wie -end beim Partizip und partizipialen Adjektiv; denn etwas ist für mich erfreulich, wenn es bewirkt, daB ich darob erfreut bin. ${ }^{18}$

Manche solcher Adjektive sind auch in der Weise systematisch polysem, daB sie gleichermaßen einen emotiven Zustand meinen können - wie z.B. traurig in den Sätzen Ich bin traurig, Er ist traurig - wie auch einen Gegenstand, der diesen emotiven Zustand kausativ bewirkt - so traurig in dem Satz Das ist eine traurige Geschichte, was bedeutet: eine Geschichte, die bewirkt, daB ich traurig werde. Ebenso ist es mit freundlich, wenn es so gebraucht wird, da $\beta$ es das kennzeichnet, was bewirkt, da $B$ ich dann freundlich bin, d.h. das, was mich freundlich stimmt, z.B. Farben oder Räume. Ähnlich wie von ergativen Verben (Er zerbricht das Glas $=$ Er bewirkt, daß das Glas zerbricht) könnte man vielleicht von ergativen Adjektiven (Das ist traurig = Das bewirkt, daß ich traurig bin bzw. werde) sprechen. ${ }^{19}$

\section{Affektive Substantive}

Mutatis mutandis gilt dies auch für Substantive und vielleicht für manche Verben. Wie wir sagen können Das ist scheußlich, was besagt: Das mutet mich in einer Weise an, daß ich mich schütteln muß vor Widerwillen; das bewirkt, daß ich mich scheußlich fühle (denn auch scheußlich ist ein systematisch polysemes Wort bezüglich Ob- und Subjektivität) ganz genauso können wir von einem Menschen etwa sagen: Das ist ein Scheusal! Und ein Scheusal ist nicht bloß (wie Adelung s.v. in dem neuen Paulschen Wörterbuch zitiert wird) eine "im höchsten Grade boshafte und lasterhafte Person", sondern vor allem auch ein (wie der neue Paul es selber formuliert) „Abscheu erregendes Wesen”, was besagt, daß es ein Wesen ist, das so beschaffen ist, daß es bewirkt, daß man bezüglich seiner voller Abscheu ist. Affektiv den Gegenpol dazu bezeichnen Wörter wie z.B. Schatz, auf Menschen angewendet. Ich erinnere mich daran, wie der Theologe Adolf Holl, in einem Spiegel-Interview gefragt, ob er nicht in dem Wiener Erzbischof und Kardinal Franz König einen ganz besonders strengen Oberhirten habe, antwortete: „Ach, der Kardinal König, der ist eigentlich ein Schatz". Das hieß nicht bloß Der meint es doch im Grunde gut mit mir, sondern auch Ich hege Zärtlichkeit für ihn. Ein Schatz ist nämlich jemand, der bewirkt, daß man bezüglich seiner Liebe oder Zärtlichkeit empfindet. 
Generell wird man von Schimpf- und Kosenamen sagen können, daß sie immer neben einer Deskriptionsbedeutung (die hier aber manchmal gar nicht ins Gewicht fällt) auch noch eine Emotionsbedeutung haben; und auf die kommt es bei diesen Wörtern an. Alle diese Wörter wären, wenn man prädikatenlogisch deuten möchte, wie die kausativen affektiven Adjektive zu beschreiben nach dem Schema $x$ bewirkt, daß ich bezüglich $x$ im affektiven Zustand $z$ bin. Und der Witz bei dem Gebrauch von Schimpf- und Kosenamen wäre, daß du, wenn ich sie in Bezug auf dich verwende, dich damit in einem affektiven Spiegel anblickst, der dir zeigt, wie du mich affizierst: ob du jemand bist, der Zärtlichkeit und Liebe einflößt oder nur Verachtung oder Haß. Daraus wäre dann die manchmal starke Wirkung dieser Schimpf- und Kosenamen zu erklären. Denn der affektive Spiegel mutet eine affek tive Selbstwahrnehmung an, wonach man hassens- oder liebenswert ist und sich also selber hassen muB bzw. lieben kann - worauf man sehr sensibel reagiert.

\section{Affektive Verben}

Also auch für Substative gilt es, daß sie, ähnlich wie gewisse Adjektive, kausativ sein können in dem hier beschriebenen speziellen Sinn. Gilt es auch für Verben? Selbstverständlich gibt es hier die auch als solche wohlbekannten kausativen Verben, die in Wendungen verwendet werden wie Das freut mich, Das tut mir leid, Das ärgert mich. Auch ihre logische Struktur ist analytisch auszubuchstabieren mittels einer Paraphrase wie: $x$ bewirkt, daß $y$ die Emotion $z$ bekommt. Hier wird aber diese Emotion direkt bezeichnet und wird außerdem auch die Person thematisch, die die Emotion hat. Weil hier alles explizit ist, handelt es sich hier um den Trivialfall kausativer affektiver Verben. Ob es aber Verben gibt, bei denen dieses kausative affektive Schema implizit ist, weiß ich nicht, mir ist bis jetzt kein gutes Beispiel eingefallen. Danach suchen könnte man am ehesten bei evaluativen Verben, wo man daran denken könnte, daß sie - jedenfalls in manchen Sprachgebräuchen - nicht allein die quasi rationale, quasi objektive Wertung, sondern auch die emotive, subjektive Reaktion auf einen Gegenstand bzw. Sachverhalt zum Ausdruck bringen, so daB also beispielsweise die Entrüstung oder die Ergriffenheit und die Bewunderung aus einer Wortwahl sprechen könnten, auch bei Verben. Etwa könnte man vermuten, daß, wenn man von einem Menschen sagt: Er säuft (im Sinne von Er ist ein Trinker), damit nicht bloB eine negative Wertung ausgesprochen ist (Er trinkt zu viel), sondern in der Regel auch Verachtung ausgedrückt wird. Dafür wäre dann die Paraphrase: $X$ verhält sich so, daß er dadurch bewirkt, daß ich im affektiven Zustand $z$ bin. 


\section{Der Affekt als Attitüde}

Wenn man es genau nimmt, muß die Paraphrase aber länger sein und lauten: $X$ verhält sich so, daß er dadurch bewirkt, daß ich bezüglich seines Tuns im affektiven Zustand $z$ bin. Das gilt analog für alle bisher angestellten Analysen kausativer affektiver Adjektive, Substantive, Verben. Immer wird mit ihnen nicht allein die Quelle, sondern eo ipso auch der Gegenstand der Affektivität bezeichnet. Nicht allein ihr Ursprung, sondern auch ihr Ziel; und diese beiden sind identisch. Wenn ich beispielsweise sage Das ist aber niedlich! - dann bekunde ich damit nicht einfach irgendein Gefühl der Zärtlichkeit und des Gerührtseins, das mich sozusagen aus dem Unbekannten anweht, sondern ein gerichtetes Gefühl. Und es ist daher auch nicht einfach so, daß ich mit diesem Satz nur zu verstehen gebe, daß ein Etwas oder Mensch bewirkt, daB ich - schlechthin gewissermaßen - zärtlich und gerührt bin. Sondern selbstverständlich sage ich mit diesem Satz zugleich auch mit aus, daB ich in Bezug auf das, was das Gefühl bewirkt, so oder so (hier: liebevoll) gestimmt bin; und nicht in Bezug auf irgend etwas anderes. Das Gefühl, mit anderen Worten, ist nichts anderes als eine affektive Attitüde. Deshalb könnte man - vielleicht präziser - auch von attitudinalen Adjektiven, Substativen, Verben sprechen, statt - was sicherlich bequemer ist - von affektiven. Allerdings ist der Begriff der affektiven Attitüde (englisch und französisch attitude), der affektiven Haltung oder Einstellung, die man zu etwas hat, sprachwissenschaftlich noch nicht etabliert. ${ }^{20}$

\section{Expressive vs. nur affektbeschreibende Vokabeln}

Soll man solche strukturellen Analysen wie die oben angestellten wirklich machen? Nun, es kann gewiß nichts schaden - wenn man nur darüber nicht vergißt, daß solche Adjektive, Substantive, und vielleicht auch Verben wie die angeführten nicht allein der Deskription von Emotionen und Gemütszuständen dienen, nicht allein der Übermittlung des Gedankens, der gewissermaßen theoretischen Erkenntnis, daß ich die Gefühle habe, die ich damit quasi diagnostisch wieder nur beschreiben, nur benennen würde. Sondern eben auch dem emotiven Ausdruck, oft sogar vor allem oder nur dem emotiven Ausdruck. Ausdruck in den Bahnen einer Konvention, es sind ja jeweils ganz bestimmte Wörter, die dem Ausdruck ganz bestimmter Emotionen dienen; deshalb eben kann man hier von lexikalisierten Emotionen sprechen. Nichtsdestoweniger jedoch unmittelbarer, echter und spontaner Ausdruck.

Jedenfalls bei einigen der angeführten Wörter; nicht bei allen. Diesen Unterschied will ich abschließend noch an einem neuen, aber wohlbekannten Beispiel deutlich machen, an dem deutschen Wörtchen arm, das wir so 
gern als Ausdrucksadjektiv verwenden. So z.B. wenn wir sagen: Ach $d u$ Arme! oder auch: Der arme Kerl! Das arme Schwein! Der arme Teufel! - ganz genauso übrigens wie man im Englischen das Wörtchen poor (Poor chap! Poor soul!) und im Französischen das Wörtchen pauvre (Le pauvre! Mon pauvre!) gebraucht. Arm ist das Wort par excellence der deutschen Sprache, das ein Mitleid ausdrückt, also nicht bloß aussagt. Diesen Unterschied beachten unsere Wörterbücher bisher noch zu wenig oder gar nicht. Denn es ist zwar gut und analytisch richtig, wenn für arm in unseren Wörterbüchern solche Synonyme angegeben werden wie unglücklich, bedauernswert, beklagenswert (DUW, s.v.). Bloß der himmelweite Unterschied von arm und diesen seinen Synonymen wird dabei nicht kenntlich. ${ }^{21}$ In der Ausdrucksdimension ist arm gerade nicht äquivalent mit unglücklich, bedauernswert, beklagenswert. Wenn ich sage: Ach, der arme Kerl! - das ist das ausgedrückte Mitleid. Dagegen wenn ich sage: Er ist bedauernswert, dann habe ich ein in der Regel kühles Urteil abgegeben.

Der Unterschied ist u.a. der, daß wir mit arm das Mitleid auszudrücken pflegen; nicht aber (oder sehr viel seltener und sicherlich auch distanzierter) mit den hier genannten Synonymen. Der Unterschied ist also, daß wir manche Wörter vorzugsweise expressiv verwenden, andere aber selten oder nie. Sie sind dazu dann auch nicht tauglich.

Das gilt ebenso auch für Adverbien. Wir pflegen ein Bedauern mit dem Wörtchen leider oder auch durch leider Gottes auszudrücken; und nicht durch bedauerlicherweise. In bedauerlicherweise liegt genau schon deshalb etwas Distanziertes, weil, wenn man dieses Wort wählt (wählt ist ausnahmsweise hier der angemessene Begriff), man damit leider abwählt und vermeidet, also dem direkten, üblichen und daher auch unmißverständlichen Ausdruck des Bedauerns ausweicht. ${ }^{22}$

Wie bereits gesagt, sind alle solche expressiven Wörter, wenn sie ausgesprochen werden, nicht auf ihre phonologische und silbische Struktur zu reduzieren, es gehört zu ihnen eine je spezifische Gestalt des nonverbalen und des parasprachlichen Verhaltens als für sie charakteristisch wesentlich dazu. Insbesondere macht auch hier die typische Intonation als angemessene melodische Gebärde die Gestalt bei solchen Wörtern mit aus. ${ }^{23}$

Leider sieht man ihre lautgestaltliche Expressivität den Wörtern in den Wörterbüchern nicht mehr an. Aber wo im Textzusammenhang das $\mathrm{Pa}$ rasprachliche und Nonverbale - wie im Schriftbild - wegfält, kann damit gerechnet werden, daB der Adressat, dem diese Wörter wirklich, aus lebendigem Gebrauch, bekannt sind, es im Wege der Gestaltergänzung 
trotzdem mithört oder mitsieht. Ohne beides kann man solche Wörter angemessen nicht verstehen. Ihr Verstehen schließt den Mitvollzug der für sie typischen Gesamtgebärde und das (sei es auch nur ansatzweise) Miterleben des in der Gebärde zu symbolischer Verkörperung gelangenden Gefühls mit ein.

\section{Intention und Präskription}

Ehe ich zu meiner zweiten Frage komme, zu der Frage nach den lexikalisierten Intentionen, muß ich nun in einem $Z$ wischenabschnitt vorbereitend etwas sagen zum Zusammenhang von Wollen einerseits und Sollen andererseits bzw. Intention und Präskription. Und erst danach werde ich dann fragen, welche Wörter dazu dienen, daß mit ihnen einem Hörer kundgetan wird, was ein Sprecher von ihm will, und ob es solche Wörter gibt und wie sie, wenn sie existieren, funktionieren.

Solche Wörter glaube ich entdeckt zu haben in Gestalt der Wörter, die - wie ich es nenne - eine präskriptive oder auch deontische Bedeutung haben. Diese Wörter, die ein Sollen mitbesagen, zeigen nämlich eo ipso auch das Wollen dessen an, der sie gebraucht. Sie können dies vermöge des grundsätzlichen Zusammenhangs, der zwischen Wollen einerseits und Sollen andererseits besteht.

Du willst, also soll ich

Ich selber bin auf den Zusammenhang von Wollen und Sollen so gekommen, daß mir ein Vers von Goethe eingefallen ist: „Und aller Wille / Ist nur ein Wollen, weil wir eben sollten, / Und vor dem Willen schweigt die Willkür stille". Wir werde aber sehen, daß der Zusammenhang auch umgekehrt besteht. ${ }^{24}$

Wir finden ihn in unserer Alltagssprache regelmäBig. Soll ich dir noch einen Kaffee machen? heiBt soviel wie: Willst du, daß ich dir noch einen Kaffee mache? Oder Sollen wir noch was bestellen? heißt: Willst du, daß wir noch was bestellen? Solche Fragen mit der Wendung Soll ich ... ? scheinen etwas höflicher zu sein als Fragen mit der Wendung Willst du, daß ich ...? In der Frage Soll ich ...? ist entgegenkommend gleich mitausgesagt, daß, wenn du es nur möchtest, ich es tun will, nach dem Motto sozusagen: Dein Wunsch ist mir Befehl. In der Frage Willst $d u$...? wird auf deinen Willen abgehoben, in der Frage Soll ich ...? wird auf meine Dienstbereitschaft abgehoben. Damit ist der Adressat der kleinen Peinlichkeit enthoben, selbst Ich will zu sagen, was sich ja nach alten Anstandsregeln nicht gehört, man sagte allenfalls: Ich möchte. Außerdem 
ist Soll ich ...? kürzer und bequemer als die Hypotaxe Willst du, daß ich ...? ? 25

Das sind aber nur Nuancen. Festzuhalten ist vor allem, daB die beiden Ausdrucksweisen, die mit Soll ich ...? und mit Willst du, daß ich ...? sprechakttheoretisch äquifunktional sind und insoweit synonym sind.

Wissen das die deutschen Wörterbücher? Noch nicht alle. Manche kennen nur den Spezialfall der Bedeutung, wo das Verbum sollen einen „Wunsch des Sprechers” ausdrückt (DUW, s.v.) mit dem Beispiel: „Du sollst dich hier wie zu Hause fühlen". Den allgemeinen Fall beschreibt jedoch das Wörterbuch der deutschen Gegenwartssprache (Klappenbach/Steinitz 1976), wenn es (s.v. sollen) gleich an erster Stelle sagt: „drückt aus, daß der Wille von jmdm., der nicht grammatisches Subj. ist, auf die Realisation des Inhaltes des Inf. gerichtet ist". Das trifft in der Tat den Nagel auf den Kopf. „N soll etwas tun” wird dort erklärt durch ,jemand will, fordert, daB $\mathrm{N}$ etwas tut". Beispiele, die dazu gegeben werden, sind u.a.: "(Sag ihr,) sie soll sofort zu mir kommen”; „Soll ich gehen?”; „Ich soll dreimal täglich eine Tablette nehmen”.

Hier haben wir in wünschenswerter Deutlichkeit herausgestellt: Des einen Sollen ist des anderen Wollen. Und $d a \beta$ der eine soll, beruht nicht nur darauf und ist nicht nur das Resultat davon, nein, es besteht darin, daß jemand anderer will. Und was der eine soll, das ist der Inhalt dessen, was der andere will. Also man könnte, Goethe variierend, sagen: Alles Sollen / Ist nur ein Sollen, weil da jemand wollte.

\section{Wessen Wollen stiftet hier das Sollen?}

Daher wird man sich bei jedem ausgesagten Sollen fragen: Wessen Wollen stiftet hier das Sollen? ${ }^{26}$ Manchmal ist es Gott, der etwas will, was wir dann sollen einfach deshalb, weil es Gott will. Denn so heißt es ja im Alten Testament, und so beginnen ja die Zehn Gebote: ${ }^{27}$ „Ich bin der Herr, dein Gott ... . Du sollst ...", die also mit dem Verbum sollen Gottes Wollen kundtun; dieses Wollen Gottes ist das kollektive Wollen, das sich die Gesellschaft in den autosuggestiven Akten des Gebetes und des Gottesdienstes sozusagen immer wieder einschärft. Manchmal ist es auch das Wollen der moralischen, der praktischen Vernunft, von dem ein Sollen ausgeht. So das absolute pflichtgemäße Sollen, das bei Kant aus dem vernunftgemäßen Wollen abgeleitet wird. „Handle nur”, heiBt es in der Grundlegung zur Metaphysik der Sitten, ,nach derjenigen Maxime, durch die du zugleich wollen kannst, daB sie ein allgemeines Gesetz werde". ${ }^{28}$ Manchmal ist es auch der Staat, der etwas will, was man dann soll, wie beispielsweise in den Versen aus dem Vormärz, die 
das von einer Obrigkeit erlassene Versammlungsverbot verspotten: „Wo dreie beieinander stehen / Da soll man auseinander gehen". ${ }^{29}$ Aber oft ist es auch einfach nur das Wollen irgendeines Sprechers, das ein Sollen eines Hörers in die Welt setzt. Putz' dir jetzt bitte noch die Zähne! - Ich mag aber heute nicht. Hör' mal, ich habe dir gesagt, du sollst dir bitte jetzt die Zāhne putzen! So oder ähnlich heiBt es hierzulande jeden Abend tausendfach.

Ich halte also fest: Das Wollen eines Sprechers wird dem Hörer oft so angezeigt, da $B$ ausgesagt wird, was der Hörer soll. Und umgekehrt kann dann der Hörer aus dem ausgesagten Sollen auf das Wollen dieses Sprechers schließen. Eine Sollensäußerung ist eo ipso eine Willensäußerung. Noch einmal etwas anders formuliert: Die ausgesagte Präskription bezüglich dessen, was der Hörer tun soll, ist nichts anderes als die ausgedrückte Intention bezüglich dessen, was der Sprecher will, daß es der Hörer tun soll.

Damit komme ich zu meinen Wörtern mit deontischer Bedeutung; also solchen Wörtern, die semantisch nicht nur deskriptiv sind, sondern ebenso auch präskriptiv sind; und die daher nicht allein ein Sein bedeuten, sondern auch ein Sollen; und die deshalb auch geeignet sind, ein Wollen anzuzeigen.

\section{Lexikalisierte Intentionen}

Gibt es solche Wörter? Nun, auch hier sind, wie schon bei den kausativen affektiven Verben, triviale Fälle von den nicht so trivialen unterscheidbar. Und die trivialen Fälle sind auch hier die expliziten. Beispielsweise ist die Pflicht (unabhängig davon, ob sie moralischer, juristischer oder dienstlicher Natur ist) Inbegriff von allem, was man tun soll; im konkreten Fall auch etwas ganz Bestimmtes, was man tun soll, so z.B. in dem Satz Das ist jetzt deine Pficht, denn er bedeutet ja konkret nichts anderes als: Das sollst du tun. Diese insbesondere Kantische (bzw., wie man fälschlich auch zu meinen pflegt, besonders preuBische) Vokabel Pficht bezeichnet zwar das je gemeinte Sollen als ein objektiv gesolltes; aber in der kommunikativen Alltagspraxis wird ein jeder Sprecher doch nur das als eines Hörers Pficht bezeichnen, was auch subjektiv der Sprecher will, $\mathrm{daB}$ es der Hörer tue. Ähnlich ist es auch mit Wörtern wie geboten und verboten oder falsch und richtig und natürlich mit den Grundvokabeln unserer Sollenssprache, mit dem Wörtchen gut und mit dem Wörtchen schlecht, wenn sie sich auf ein Tun beziehen. Gut ist dann ja, was man (bzw. wie man etwas) tun soll, und schlecht ist, was man (bzw. wie man etwas) nicht tun soll. Alle solche Wörter können evidentermaßen dem Appell an einen Adressaten dienen. 
Außer solchen trivialen Fällen gibt es, wie gesagt, die nicht so trivialen, also Wörter, die ein Sollen mitbesagen, aber ohne daß dies ohne weiteres bewußt ist.

\section{Wörter mit deontischer Bedeutung}

Unkraut ist ein solches Wort, mit dem ein Sollen ausgesagt wird; es ist mein Standardbeispiel. Mit dem schlichten Satz Das ist ein Unkraut sagt man aus: Das darf man, ja das soll man ausreißen oder sonst irgendwie vernichten. Das ist, wie ich meine, integrierender Bestandteil der Gesamtbedeutung dieses Wortes. Unkraut hat auch eine Deskriptionsbedeutung. Denn Unkraut heiBen „Pflanzen, die zwischen angebauten Pflanzen wild wachsen [u. deren Entwicklung behindern]" (DUW, s.v.), also anderen Pflanzen schaden. Aber mitgesagt in Unkraut ist auf alle Fälle auch: Das muß hier weg. Oder: Das gehört hier ausgerissen und vernichtet. Und in dieser präskriptiven Komponente der Bedeutung, im deontischen Bedeutungsanteil, liegt, so meine ich, die Pointe dieses Wortes.

Ähnlich ist es auch bei Ungeziefer, was nicht einfach nur rein deskriptiv ein Wort für "[schmarotzende] tierische Schädlinge” ist, z.B. "Flöhe, Läuse, Wanzen, Milben, Motten, auch Ratten und Mäuse" (DUW, s.v.) - also, füge ich hinzu, für solche kleinen Tiere, die typischerweise massenweise da sind und vor denen es dann nur so wimmelt, wie wir sagen; und vor denen man, so füge ich des weiteren hinzu, sich als ein normal sozialisierter Zeitgenosse ekelt (dieses ist der emotive-expressive Anteil der Bedeutung). Sondern Ungeziefer, das bedeutet außerdem auch präskriptiv, genau wie Unkraut, etwas, was man zu vertilgen, zu vernichten hat. Und darin liegt auch das Perfide, wenn man Menschen metaphorisch Ungeziefer nennt, wie das ja in der jüngeren Geschichte immer wieder vorgekommen ist. Denn das bedeutet nicht-metaphorisch u.a.: Das sind keine Menschen, sondern Tiere, das sind Schädlinge, vor denen man sich ekelt, und vor allem heißt es: Diese Menschen soll man deshalb töten und vernichten. Die deontische Bedeutung bleibt erhalten im uneigentlichen, metaphorischen Gebrauch des Wortes.

Das ist sicherlich ein drastisches Exempel, und ich füge noch ein anderes drastisches hinzu. Carl Améry erzählt, wie er in Wien im Jahre 1935 im Kafteehaus in der Zeitung den Text der Nürnberger Gesetze las. Und er sagt, da wurde ihm auf einmal klar: Erstens, ich bin ein Jude, was er vorher seinem Selbstverständnis nach nicht war. Und zweitens, so schreibt er wörtlich: „Jude sein, das hieß für mich von diesem Anfang an, ein Toter auf Urlaub sein, ein $z u$ Ermordender ...". ${ }^{30}$ In der Tat war 
das für die Partei von Hitler vor wie nach den Nürnberger Gesetzen die deontische Bedeutung des Wortes Jude.

\section{Präskriptionsbedeutung, Gerundivbedeutung}

In der lexikalischen Semantik ist der Terminus deontisch noch nicht üblich, und es fragt sich, ob man eine andere Bezeichnung wählen sollte. Statt von der deontischen Bedeutung kann man auch von einer präskriptiven oder Präskriptionsbedeutung oder Präskriptivbedeutung reden. Allgemeinverständlich könnte man auch sagen: Sollensbedeutung. Müller (1993, S. 21) nennt den Spezialfall der deontischen Bedeutung, der im folgenden erläutert wird, gerundive Bedeutung. Dafür werde ich hier $\mathrm{Ge}$ rundivbedeutung sagen. ${ }^{31}$

Wir erinnern uns aus unserer Schulzeit an das Gerundivum. Das ist jenes im Lateinischen vorhandene verbale Adjektiv, das - attributiv oder prädikativ verwendet - mit prägnanter Kürze aussagt: das ist etwas, was ge-x-t werden soll, wenn $x$ der Verbstamm ist. Denn a) Passiv und b) Sollen sind die zwei Bedeutungskomponenten, die das Gerundivum definieren. Als Prädikativum ist das Gerundiv bequem zu übersetzen. Puer est laudandus heißt auf deutsch: Der Knabe ist zu loben (was in diesem Satz nicht heißt Man kann ihn, wenn man möchte, loben, sondern: soll ihn loben). Schrecklich unbeholfen klingt im Deutschen nur die Schulbuchübersetzung für das Gerundiv als Attribut. Aus einem puer valde laudandus wird dabei ein sehr zu lobender Knabe. Im Französischen, im Italienischen, im Englischen läßt sich das oft viel eleganter sagen. Un livre à lire $\mathrm{z} . \mathrm{B}$. ist genauso wie un libro da leggere und a book to be read dasjenige, was auf deutsch ein zu lesendes Buch ist, d.h. eines, das gelesen werden soll. Also die deontische Bedeutungskomponente sollen ist im Gerundivum eingebaut in die Bedeutung.

Was im Gerundiv grammatikalisiert ist, eben die deontische Bedeutung, kann jedoch auch lexikalisiert sein, was bei vielen Wörtern in der Tat der Fall ist. Beispielsweise in den gerundiven Adjektiven, wie ich sie hier deshalb nennen möchte.

\section{Gerundive Adjektive}

Eine Durchsicht unseres Standardwerks zur Wortbildung des deutschen Adjektivs (Kühnhold/Putzer/Wellmann 1978) ergibt für meine Zwecke zweierlei Befunde: a) es haben manche Gruppen der dort aufgeführten Adjektive eine Gerundivbedeutung; b) die Autoren haben dafür keinen Terminus, verwenden aber (S. 474) die Bedeutungscharakterisierung, da $B$ in solchen Adjektiven „etwas (in der Basis ausgedrückt) als 
erwünscht, günstig oder nötig erscheint" und sprechen diesbezüglich (per Kapitelüberschrift) von einem „Ausdruck der Empfehlung”.

Unter diesem Titel werden dort (S. 474-478) behandelt:

- Adjektive auf - wert, z.B. lesenswert (mit dem Beispiel „lesenwertes Buch” und der Bedeutungsparaphrase „Buch, das gelesen werden sollte"); nachahmenswert; sehenswert; fluchenswert; hassenswert; erwähnenswert (was „zu erwähnen ist/erwähnt werden sollte/erwähnt zu werden verdient”); lobenswert (mit dem Beispiel „lobenswerter Fleiß” und der Erläuterung „Fleiß, der zu loben ist/gelobt werden sollte"); ${ }^{32}$

- Adjektive auf -würdig, z.B. vertrauenswürdig ("vertrauenswürdige Person": „Person, der man Vertrauen schenken sollte/kann”); bewundernswürdig; förderungswürdig. Allgemein wird dazu angemerkt: „Die modale Leistung reicht vom 'können' (glaubwürdige Darstellung, abbauwürdige Kohle) über die zentrale Bedeutung 'sollen' (erhaltungswürdige Gebäude, vertrauenswürdige Person) bis zu 'müssen' (fluchwürdige Tat, verabscheuungswürdige Bluttat)";

- Adjektive auf -bedürflig, z.B. revisisonsbedürfig („revisisonsbedürftiges Programm": „P., das (dringend) revidiert werden sollte”); geheimhaltungsbedürftig; renovierungsbedürftig; reparaturbedürftig; korrekturbedürftig;

- Adjektive auf -reif, z.B. schrottreif („schrottreife Gewehre”: „die (bald) verschrottet werden sollten"); abbruchreif ("abbruchreifes Haus”: „Haus, das bald abgebrochen werden sollte”).

Fazit: Gerundive Adjektive gibt es zahlreich, und mithilfe produktiver Adjektivsuffixe und -affixoide wie -wert, -würdig, -bedürftig, -reif ist ihre Zahl beliebig zu vergrößern.

Außerdem gibt es auch manche offenbar als solche noch bis heute nicht erkannten gerundiven Adjektive. Beispielsweise achtbar, das nach Kühnhold/Putzer/Wellmann (1978, S. 391) angewendet werden kann auf $\mathrm{El}$ tern. Sind achtbare Eltern solche Eltern, die man achten kann, wenn man das möchte, wie hier durch die Reihenbildung (denkbar, deutbar, faßbar, hörbar usw.) und durch eine Formel - mit poss (für möglich) als Bedeutungsindikator - ausgesagt wird? Nein, offensichtlich sind es Eltern, die geachtet werden sollen. Überhaupt hat -bar nicht selten eine gerundivische Bedeutung. So ist zahlbar selbstverständlich nicht, was man bezahlen kann, sondern, wenn in einer Rechnung steht, zahlbar bis zum 1.5.1999, dann bedeutet das, daß man bis dahin zahlen soll. So ist strafbar eine Handlung, die bestraft werden soll (nicht kann). Ein Zufallsfund 
belehrt mich, daB (in fachsprachlichem Spezialgebrauch) sogar verwertbar gerundivisch sein kann: „Im Vergleich zu 1991 haben sich die thermisch verwertbaren Siedlungsabfalle, das heißt alles, was noch verbrannt werden muß, 1992 um 9000 Tonnen vermindert" (Heidelberger Amtsanzeiger 25.2.1993). Demnach wäre neben poss alternativ auch noch necess bzw. obligat als Indikator in die Formel der semantischen Struktur von -bar-Adjektiven einzusetzen.

Ähnlich steht es auch mit Adjektiven auf die Endung -lich. Löblich etwa ist ein Synonym von lobenswert, und es bedeutet gleichfalls was gelobt werden soll. Sträflich ist, was (eigentlich) bestraft gehört. Vertraulich ist eine Information, die mit Diskretion behandelt werden soll. Gefährlich ist, was in Gefahr bringt und wovor man sich daher in acht nehmen soll (wovor sich gehütet werden soll).

An diesem letzten Beispiel scheiden sich, wie ich aus Diskussionserfahrung weiB, die Geister. Anhänger einer Minimalsemantik wollen nach wie vor nur (nebst dem genus) eine (genau eine) differentia specifica (den distinguisher) als konstitutiv für die Bedeutung eines Wortes gelten lassen. Sie bestreiten nicht, daB eine Äußerung wie Das ist gefährlich sprechakttheoretisch eine Warnung sein kann und als solche dann pragmatisch mit Davor muß man sich vorsehen! und mit Vorsicht! äquifunktional ist; nur erklären sie dies durch Implikatur. Prototypsemantiker dagegen sind daran gewöhnt, Bedeutung als den Inbegriff von allem zu verstehen, was bei der Verwendung eines Wortes in der Regel (per default) mitgedacht und mitgemeint wird, und sie nehmen also keinen Ansto $B$ daran, da $\beta$ Bedeutungsparaphrasen eine Wortbedeutung so bestimmen, da $\beta$ sie logisch (nach der klassischen Vorschrift für Definitionen) überdefiniert ist.

Akzeptiert man dies als Möglichkeit, dann wird man auch bereit sein, etwa schuldig (in der einen der Bedeutungen des Wortes) so zu deuten, daß es nicht allein bedeutet "(an etwas) die Schuld tragend, in bezug auf jemanden, etwas Schuld auf sich geladen habend" (DUW, s.v.), sondern außerdem auch mitbedeutet: und deshalb zu bestrafen. Denn wir wissen ja: Strafe muß sein, wenn jemand schuldig ist. Daß jemand schuldig ist, rechtfertigt nicht allein, nein, es verlangt danach, daß er bestraft wird. Und dies ist in der "Vorstellung", wie man früher sagte, mitenthalten, die man von jemand hat, der schuldig ist; heute würde man computermetaphorisch sagen können, daß schuldig und zu bestrafen eng "vernetzt" sind. Beides läuft jedoch darauf hinaus, $d a ß$ die deontische Bedeutung $z u$ bestrafend zur Gesamtbedeutung dieses Wortes (schuldig) beiträgt. 


\section{Gerundive Substantive}

Gerundive Substantive sind die oben schon genannten Unkraut sowie Ungeziefer, aber das sind Wörter, wo die Gerundivbedeutung implizit ist. Für die Skeptiker bezüglich eines in Lexemen ausgesagten Sollens überzeugender sind sicherlich auch hier (beim Substantiv) die expliziten Fälle solcher Wörter, die die Lehnmorpheme -andum oder -and enthalten und die leicht zu finden sind anhand des Wörterbuchs von Muthmann (1988, S. 551 und S. 169-171). Unzweifelhaft ist wohl ein Explikandum etwas, was zu explizieren ist, d.h. expliziert werden soll, ein Definiendum das, was definiert werden soll; und ähnlich ein Analysand, Habilitand, Konfirmand, Examinand jemand der respektive analysiert, habilitiert, konfirmiert, examiniert werden soll. Ganz genauso wie ein Auszubildender-sogar auch dann noch, wenn er sich auf den $A z u b i$ reduziert hat jemand ist, der ausgebildet werden soll. ${ }^{33}$

Wie schon bei den Adjektiven ist es aber auch bei Substantiven schon von vornherein wahrscheinlich, $\mathrm{da} B$ es die Bedeutungskomponente sollen (oder werden sollen) nicht allein bei Wörtern gibt, die diesbezüglich motiviert sind, sondern auch bei anderen Wörtern, wo die Gerundivbedeutung also implizit ist. Ähnlich, wie in manchen Blumennamen explizit gesagt ist, daß es sich beim Denotat um eine Blume handelt (so bei Sonnenblume, Dotterblume, Kornblume), nicht jedoch in anderen Blumennamen (so bei Rose, Tulpe, Akelei), bei denen es jedoch nichtsdestoweniger genauso wesentlich für die Bedeutung ist, daß diese Pflanzen Blumen sind, statt irgendwelche anderen Pflanzen. Und wir finden solche Substantive - mit deontischer, mit gerundivischer Bedeutung - in der Tat sogar in unseren Wörterbüchern als deontisch ausgewiesen. Hier in bunter Reihenfolge einige Exempel.

So ist eine Einbahnstraße eine „Straße, die nur in einer Richtung befahren werden darf' (DUW, s.v.); nicht etwa: wird bzw. kann. So erklärt es sich, daß man beim Autofahren eine Äußerung wie Das ist eine Einbahnstraße nicht als die gewissermaßen wertneutrale Darstellung (im Sinne Bühlers) eines Sachverhalts versteht, sondern als Warnung oder (negative) Aufforderung: Fahr da nicht aus Versehen rein! Das Sollen (oder negativ: Nicht-Dürfen) ist in der Bedeutung schon des Wortes mitenthalten.

Eine Miete ist der "Preis, den man für das Mieten von etw. ... zahlen muB" (DUW, s.v.). Also das Sollen steckt auch hier in der Bedeutung dieses Wortes mit darin, denn eine Miete, die man gar nicht zahlen müßte, wäre keine, sondern ein Geschenk an den Vermieter. 
Ähnlich ist die Steuer ein "bestimmter Teil des Lohns, Einkommens oder Vermögens, der an den Staat abgeführt werden muf" (DUW, s.v.); und sind Schulden ein „Geldbetrag, den jemand einem anderen schuldig ist" (DUW, s.v.), und hier bedeutet schuldig, daB jemand ,zu geben verpflichtet" (DUW, s.v.) ist. Die Rechnung ist eine "schriftliche Aufstellung ... mit der Angabe des Preises, der ... zu zahlen ist" (DUW, s.v.). Offensichtlich ist die Gerundivbedeutung häufig anzutreffen, wo es um das Geld geht.

Ein Ziel ist a) ein Punkt oder Ort, „bis zu dem man kommen will ...” oder b) „etw., was beim Schieben, Werfen o..̇. ... getroffen werden soll' oder c) etwas, ... was man ... als angestrebtes Ergebnis seines Handelns, Tuns zu erreichen sucht" (DUW, s.v.): man sieht hier, wie in den Bedeutungsparaphrasen will und soll (mutatis mutandis) dieselben Dienste tun. In einer sprechakttheoretischen Studie wird definiert: „Ziele sind ... Weltzustände, die ... herbeigeführt werden sollen" (Motsch/Pasch 1987, S. 23).

Eine Arbeit ist - in einer der Bedeutungen des vielfach polysemen Wortes - wie die alten (aber nicht die neuen) deutschen Wörterbücher wissen, nicht nur "dasjenige, was durch Arbeit hervor gebracht worden [ist]", sondern ebenso auch "dasjenige, was durch die Arbeit hervor gebracht werden solP" (Adelung $(1808$, s.v.); ähnlich definieren Arbeit Campe (1807) und noch Sanders (1860): „der Gegenstand, das Werk der arbeitenden Thätigkeit, und $z$ war sowohl das geschaffte als das zu schaffende". Also unsere alten Wörterbūcher waren noch sensibel für den Unterschied, ob eine Arbeit schon getan ist oder noch zu tun ist. Aber Arbeit ist auch heute noch (u.a.) das, was getan werden soll. Denn nur so ist es erklärlich, daß, wenn man zu jemand sagt Das ist deine Arbeit, dieser Satz soviel bedeuten kann wie Das sollst du machen oder Mach das!

Diese kleine Liste gerundiver Substantive soll hier nur plausibel machen, daB es so etwas tatsächlich gibt wie die deontische Bedeutung, hier im Spezialfall einer Gerundivbedeutung. Und daß sie, nicht immer, aber oft, durchaus auch in den Wörterbüchern ausgewiesen wird. Auf die wirklich, wie ich finde, interessanten Fälle einzugehen, ist hier nicht der Ort: die großen Wörter insbesondere der sozialen und politischen BewuBtseinsbildung wie Nation bzw. Deutschland (beides hierzulande lange das, was noch geschaffen werden sollte), Demokratie (im 19. Jahrhundert eine Staatsform, die man je nach Standpunkt und nach Gruppensprache schaffen oder auch verhindern sollte), Sozialismus (als Gesellschaftsform, die in der DDR aufgebaut werden sollte, bis man dann befunden hat, sie sei schon existierend). Oder Wörter der moralischen BewuBtseinsbildung wie das eben schon genannte Arbeit und das Verbum arbeiten. "Wer nicht arbeitet, soll auch nicht essen" - dieser Satz ist, wie ich meine, prägend nicht nur für das europäische und deutsche Denken, sondern auch für die Semantik - wenn auch nicht die Minimalsemantik - dieses Wortes. ${ }^{34}$ 
Zur Infragestellung des Begriffs 'Proposition' in linguistischem Gebrauch

Wenn ich richtig sehe, hat die Linguistik bisher Wörter mit deontischer Bedeutung als Faktoren des Zustandekommens des illokutionären Sinnes eines Satzes kaum beachtet, und sie hat sich bei der Suche nach Illokutionsindikatoren auf Grammatisches (im alten Sinn von: was in der Grammatik abgehandelt wird, und nicht im Wörterbuch) beschränkt, zumindest konzentriert. So vor allem auf den Modus, Modalverben, Wortstellung, Intonation, Akzent. Aus der (i.e.S.) Lexik gelten nur die Satzadverbien und -adverbiale als für den illokutionären Sinn des Satzes wichtig. Substantive, Adjektive, Verben mit deontischer Bedeutung werden als konstitutive Elemente der Erzeugung illokutionären Sinnes bisher außer acht gelassen.

Dabei zeigen gerade solche Wörter oft den illokutionären Sinn an, den ein Satz hat. Das so oft zitierte Beispiel für den indirekten Sprechakt - Es zieht! - ist in seinem illokutionären Sinn - Mach bitte schnell die Tür oder das Fenster $z u$ ! - doch wohl nur zu verstehen, wenn man weiB, was jedes Glied der deutschen Kommunikationsgemeinschaft weiB: daß es nicht ziehen darf und daß ein Zug, wenn er entsteht, behoben werden soll, besonders wenn es kalt ist; hingegen ist ein leichter Lufthauch in der Wohnung etwas Angenehmes und Erwünschtes, wenn es heiß ist. ${ }^{35}$ Wenn in einem Satz von einem lesenswerten Buch die Rede ist, dann weiß genauso jeder Sprecher unserer Sprache, daß er damit aufgefordert ist (ihm angeraten wird), das Buch zu lesen. Und wenn etwas Unkraut ist, dann weiß man gleichfalls, was damit geschehen soll bzw. sollte. Dazu noch als letztes Beispiel eine Lesefrucht aus einem Buch von Horvath (Jugend ohne Gott), wo der Autor einen Bauern über eine Diebesbande obdachloser Kinder sagen läßt: „Denen trau ich alles zu. Es ist Unkraut und gehört vertilgt!" ${ }^{36}$ Wie ich diesen zweiten Satz verstehe, meint er: Es ist Unkraut und gehört aus diesem Grund vertilgt. Denn Unkraut ist nun einmal, ganz bestimmt für einen Bauern, so geartet, daß man es vertilgen soll, wo man es antrifft, und das bleibt, wie schon gesagt, erhalten auch bei metaphorischer Verwendung dieses Wortes. Also alle diese Sätze haben den Appellcharakter, den sie haben, weil die Wörter, die darin gebraucht sind, ihrem Hörer sagen, was er soll. Was er, wie man allerdings genauer unterscheiden müßte, erstens wollen soll und zweitens, wenn dies möglich ist, auch tun soll. ${ }^{37}$ Damit aber eo ipso gleichfalls sagen, was der Sprecher will, daß es der Hörer wollen oder tun soll.

$\mathrm{DaB}$ man in der Linguistik dafür bislang blind ist, liegt, wie ich vermute, wesentlich auch daran, daß die Linguistik aus der Logik - unbesehen sozusagen - den Begriff Proposition entlehnt hat, der semantisch eindimensional ist. Denn eine Proposition kann weder emotiv sein noch 
deontisch, sondern bloß wahr oder falsch. So ist sie nämlich definiert: es wird, wenn wir den Terminus Proposition auf einen Satz anwenden, systematisch abstrahiert von allem, was zu dessen Wahrheitsfähigkeit nichts beiträgt. In der Prädikatenlogik wird auf diese Weise etwa aus dem Satz Das ist gefährlich ein deontisches und affektives Neutrum, das als solches keine Angst zum Ausdruck bringen kann und das auch nicht besagt, daB hier mit einer Sache oder einem Sachverhalt vorsichtig umgegangen werden soll. Dieser Satz ist daher für die Logik auch nur die Behauptung der Proposition, daß das gefährlich ist, und insbesondere ist er keine Warnung, was er aber doch normalerweise ist, wie oben schon gesagt: pragmatisch ist er ja geradezu äquivalent mit Vorsicht! Nicht, daß dies die Logiker - gewissermaßen als Privatpersonen - gar nicht wüßten. Aber für die Prädikatenlogik und Aussagenlogik, die sich bloß für Folgerungsbeziehungen von Sätzen interessiert, für die es nur auf Wahrheitswerte ankommt, ist dies in der Tat nicht relevant. Und so reduziert sich in der logischen Betrachtung die Bedeutung eines Satzes wie Das ist gefährlich darauf, daß hier das Bestehen eines Sachverhalts behauptet wird. Und die Bedeutung eines Wortes wie gefährlich reduziert sich darauf, daß es eine kognitive Eigenschaft bedeutet, also etwas darstellt. Nicht jedoch auch etwas ausdrückt und signalisiert, im Sinne Bühlers. Der Begriff Proposition macht alles unsichtbar, was in der Satzund Wortbedeutung Ausdruck und Appell ist, und das soll er auch, denn dazu hat man ihn gebildet. ${ }^{38}$

Für die Linguistik ist daher der Terminus Proposition ein zweifelhaftes Erbe aus der Logik. Soll man also in der Linguistik auf den Terminus verzichten? Oder soll man ihn in neuer Weise derart definieren, daß er emotive und deontische Bedeutungskomponenten nicht mehr ausschließt? Diese beiden Möglichkeiten gibt es.

\section{Wirklichkeit als kognitiv und emotiv und präskriptiv geprägte}

Wie ich hoffe dargetan zu haben, gibt es wirklich Wörter, die im Sinn von Bühlers Drei-Funktionen-Schema multifunktional sind: Wörter, die, in Personalunion, die Rollenträger sind von deskriptiven und auch expressiven Elementen der Bedeutung; oder Träger sind von deskriptiven und auch präskriptiven Elementen der Bedeutung; oder Träger sind von deskriptiven und auch expressiven und auch präskriptiven Elementen der Bedeutung. Gibt es Gründe für die Existenz von solchen sozusagen Doppel- oder Dreifachwörtern? Ungeziefer war ein solches Wort, das deskriptiv und emotiv und präskriptiv ist. Das ist ökonomisch. Solche Wörter schlagen ja, wenn man so will, zwei Fliegen oder drei mit einer 
Klappe. Ich vermute aber, daß es auch noch einen anderen - den eigentlichen, wie ich meine - Grund der Existenz von solchen Wörtern gibt. Dieser Grund ist, wie ich meine, ontologisch.

In der Bühlerschen Ontologie sind dargestellte Wirklichkeit und ausgedrückte Emotion und mitgeteilte Intention vollkommen separiert, sie haben darin miteinander nichts zu tun. Darstellung ist für Bühler immer emotionslos und appellfrei. Das bedeutet aber auch: die Wirklichkeit der Gegenstände und der Sachverhalte - ist für Bühler eine emotiv und präskriptiv neutrale. Eine Wirklichkeit, in der kein Unkraut, nichts Erfreuliches und nichts Entsetzliches und keine Einbahnstraßen existieren. Bühlers Wirklichkeit ist offensichtlich eine szientifisch-wissenschaftliche. Ihre Gegenstände/Sachverhalte haben keine affektiven, keine präskriptiven Eigenschaften, sondern nur die kognitiven, die mit dem Begriff Proposition gemeint sind.

Wir leben aber alle nicht in einer Welt, die, wie der junge Wittgenstein $(1921$, S. 1) gesagt hat, nichts weiter ist als „alles, was der Fall ist". Denn wir stehen Gegenständen oder Sachverhalten, von Personen ganz zu schweigen, wenn wir sie betrachten und erleben, nicht neutral, gewissermaßen wissenschaftlich, gegenüber. Wie auch sie nicht uns. Die Gegenstände, Sachverhalte und Personen muten uns auf ganz bestimmte Weisen an und fordern uns zu ganz bestimmten Weisen des Verhaltens auf. Manche Menschen sind sympathisch, manche Sachverhalte dieser Welt sind unannehmbar. Dazu möchte ich aus Überzeugung sagen: Sie sind wirklich unannehmbar (was bedeutet: man darf sie nicht akzeptieren) oder auch sympathisch (was bedeutet, daß wir für sie Sympathie empfinden). Das gehört zur Wirklichkeit dazu, in der wir leben, wenn auch nicht zur Wirklichkeit der Wissenschaften. Denn als kognitiv und emotiv und präskriptiv geprägte Wirklichkeit erleben wir die Welt, und daher zeigt sich uns die Welt auch so in unserer Sprache, die jedoch auch umgekehrt das Ihre dazu beiträgt, wie die Welt geprägt ist, kognitiv und emotiv und präskriptiv. 


\section{Anmerkungen}

2 Konnotation ist ein Begriff, den ich im folgenden vermeide. Erstens und vor allem wegen der ihm inhārenten Abwertung der Wichtigkeit der emotiven (und der präskriptiven) Komponenten der Bedeutung von Lexemen; Dieckmann (1979, S. 112) gibt zu recht als Hauptbedeutungsmerkmal von Konnotation in zeitgenössischer Verwendung an: „nicht oder nur am Rande zum Gegenstandsbereich der lexikalischen Semantik gehörig" (aus demselben Grunde werde ich auch den Begriff Gefühlswert (Erdmann 1900) nicht verwenden). Zweitens wegen seiner Mißverständlichkeit; Dieckmann (1979, S. 100) nennt den Diskussionsstand „schlicht chaotisch”. Zwar hat Rōssler (1979) den Versuch gemacht, Konnotation durch Klärung des Begriffs zu retten, aber das Ergebnis des Versuchs fällt, scheint mir, so aus, daB man nun den Terminus erst recht nicht mehr verwenden möchte. Einen Überblick über die jūngere Diskussion gibt Ludwig (1991, S. 5ff.).

3 Wenn ich Kognition und Emotion und Intention die Zeichendimensionen nenne, knüpfe ich an Bühler (s.u.) an, der in Bezug auf seine Trias von Darstellung, Ausdruck und Appell selber von den Dimensionen - und zwar Sinndimensionen - des sprachlichen Zeichens gesprochen hat (vgl. Ammann 1988, S. 55). Mit Verweis auf Būhler redet auch Max Black (1973, S. 153ff.) von drei Dimensionen der Sprache, die er als die präsentative, expressive und dynamische bezeichnet. - Diese Zeichendimensionen sind nicht zu verwechseln mit den (im Anschluß an Morris 1938) manchmal (so von Vigener 1978) so genannten) semiotischen Dimensionen (nämlich Syntax, Semantik und Pragmatik).

4 Die Begriffe der Gestaltbereinigung und der Gestaltergänzung bilde ich, obwohl hier (1934, S. 28) Bühler offensichtlich den Begriff Gestalt bewußt vermeidet - sehr wahrscheinlich deshalb, weil er sich gegen die „Berliner Gestaltpsychologie” mit ihren „prinzipienmonistischen Neigungen” (Bühler 1934, S. 56) abgrenzen will. Heute haben wir ein solches Abgrenzungsbedürfnis nicht mehr, und die Linguistik kann von der Zurkenntnisnahme der gestaltpsychologischen Einsichten nur gewinnen, wie besonders Lakoff/Johnson (1980) zeigen; man vgl. dazu Liebert (1992, S. 12ff.), der auch einen Überblick über die "Gestaltgesetze" gibt und auf die neuere gestaltpsychologische Literatur verweist.

5 Wenn man hier den Test von Keller (1977, S. 9ff.) und Lang (1983, S. 333ff.) verwendet, sieht man, daB bei Nichtbestehen dieses Sachverhaltes man dem Sprecher nicht den Vorwurf einer Lüge, sondern einer Täuschung machen würde; das bestätigt, daß der Modus des mit-ausgesagten Sachverhalts im Fall des Hilferufes nicht der Modus der Behauptung ist. Gleichwohl handelt es sich aber dann um eine Täuschung in Bezug auf Gegenstände/Sachverhalte, also in der Zeichendimension der Darstellung im Sinne Bühlers. Hier von einer Präsupposition zu sprechen, ist zu wenig, denn im Hilferuf wird nicht bereits (gewissermaßen als bekannt) vorausgesetzt, daß eine Notlage besteht; deren Bestehen wird ja erst durch den Hilferuf selbst kundgetan.

6 Bühler (1934, S. 28) sagt vom Zeichen als Symptom bzw. Ausdruck nur, es sei ein solches Zeichen "kraft seiner Abhängigkeit vom Sender, dessen Innerlichkeit es ausdrückt". Zu Innerlichkeit merkt er an anderer Stelle (1933, 
S. 110) an, daß dazu "nicht nur aktuelle Erlebnisse, sondern der Inbegriff aلl der Momente gehören, aus denen Klages den Charakter logisch aufbaut". Demgegenüber wird im folgenden als Ausdruck nur der Ausdruck einer Emotion (eines Gefühls) verstanden.

$7 \mathrm{Zu}$ „Verstehen als Erkennen” vgl. Hermanns (1987); dort ist der Versuch gemacht zu zeigen, welcherlei Erkennen - außer dem von signifiant und signifié - sonst noch als Verstehen gelten kann.

8 Nicht gerade glücklich ist die Übersetzung von signifié durch Bezeichnetes deshalb, weil das Wortpaar Bezeichnendes/Bezeichnetes einem NomenklaturVerstāndnis von Bedeutung Vorschub leistet, das die Lommelsche Ūbersetzung des Cours von de Saussure auch sonst immer wieder nahelegt (vgl. Hermanns 1992).

9 So ja die berūhmte Formulierung im "Tractatus": „Einen Satz verstehen, heißt, wissen was der Fall ist, wenn er wahr ist" (Wittgenstein 1921, 4.024).

10 Die hier nur skizzierte Interpretation sowie Kritik des Bühler-Schemas weiter auszuführen ist hier nicht der Ort; zur Kritik verweise ich auf Kubczak (1984), Ammann (1988), Busse (1975), Ortner (1992), v. Polenz (1974).

11 Als Gefühlswörter bezeichnen solche Wörter Jäger/Plum (1988), (1989), die demgemäß Gefühl "synonym zum Lexem Emotion als Hyperonym für Wörter wie Liebe, Furcht, Angst, Freude etc." gebrauchen (1988, S. 37). Ähnlich sind für Alston (1967, S. 479) emotion-terms die Wörter fear, anger, indignation, remorse, embarrassment, grief, distress, joy, craving, disgust; und für Kövecses (1990) sind emotion concepts Wörter wie anger, fear, pride, respect und (romantic) love. Wenn sich auch ihre Definitionen von Gefühl erheblich unterscheiden, sind die Wissenschaftler im Gebrauch des Wortes trotzdem ziemlich einig. Ein spezielles (und komplexes) der Gefühlswörter des Deutschen hat bezüglich seiner historischen Semantik Teubert (1991) untersucht.

12 Sprechakttheoretisch unterscheidet in der deutschen Linguistik erstmals Keller (1977) zwischen dem, was man mit einem Sprechakt "sagt", und dem, was man damit "zum Ausdruck bringt", und insbesondere zwischen "Nennen von Haltungen” und „Zum-Ausdruck-Bringen von Haltungen” (S. 13). Daß eine Einstellung, "mit der" ein Sprecher etwas sagt und die er ("präreflexiv") ausdrūckt etwas anderes ist als eine Einstellung, „über die" er etwas sagt, betont auch Lang (1983, S. 329), der sich (S. 307) der „Hilfskonzepte” SAY vs. EXPRESS bedient, um den „propositionalen Inhalt" eines Satzes von der in ihm ausgedrückten "Einstellung” zu eben diesem Inhalt scharf zu unterscheiden. Fiehler (1990, S. 96ff.) stellt mit ähnlicher Pointe die „Erlebensthematisierung” bzw. die „Emotionsthematisierung” dem „Erlebensausdruck” bzw. dem „Emotionsausdruck” gegenüber und charakterisiert (S. 17) den Emotionswortschatz als "Menge der Wörter, die denotativ Erlebensformen und Emotionen bezeichnen", "im Gegensatz zur emotionalen Konnotation”. Fiehler ist mit seinem Buch zu "Kommunikation und Emotion" der Pionier der deutschen Linguistik zur Thematik Emotion und Sprache. Dabei geht es ihm jedoch vor allem um die „Rolle von Emotionen in der Interaktion" (S. 2), was bedeutet, daB er Emotionswortschatz und expressive Lexik ("lexikalisierte Emotionen") systematisch nicht behandelt. 
Als „Äußerungsformen, die ... starke emotionale Beteiligung signalisieren”, zählt er (S. 127) aber auf: „Affektlaute” (z.B. Au!), „Interjektionen” (Ohhh!), „Bewertungen (mit Ausdruck) (Herrlich!), „Manifestationen der Überraschung und des Unglaubens" (Waas?), "Beschimpfungen" (Du Trottel!), „Koseformen” (ohne Beispiel), „Flüche” (Herr Gott noch mal!), "Drohungen” (Na warte!), "Empörungen” (Jetzt reicht's!). - Gegenūber der von Keller, Lang und Fiehler als grundlegend angesetzten Unterscheidung zwischen Ausdruck und Beschreibung des eigenen Gefūhls des Sprechers meldet Alston (1967, S. 490) Skepsis an: „No one has succeeded in giving an analysis of expressing feelings (as a linguistic activity) which would sharply differentiate it from asserting that one has a feeling". Daß die Unterscheidung in der angelsächsischen Literatur schon eine längere Geschichte hat, ist am Erscheinungsdatum (1967) von Alstons Lexikonartikel zu erkennen.

13 Lexikographisch ist dies Beispiel als ein Glücksfall anzusehen, weil es einen Text zitiert, der nicht nur ein Beleg für einen Wortgebrauch ist, sondern in der Sozialisation von Generationen deutscher Kinder sicherlich der Text gewesen ist, an dem sie den Gebrauch des Wortes pfui, wie auch des Wortes garstig, exemplarisch lernten, dies im Zuge einer Reinlichkeitserziehung, die sie eo ipso auch den Abscheu und den Ekel vor dem Schmutzigen und Ungepflegten lehrte (lange Haare, Bārte, Fingernāgel usw.). Man erkennt daher an diesem Beispiel außerdem: Auch pfui hat eine kognitive Komponente. Denn es zeigt ja, daß zur Einübung des richtigen Gebrauchs von pfui dazugehört, das Kind zu lehren, in bezug auf welche Gegenstände/Sachverhalte (und Personen) pfui zu sagen angebracht ist. Auch Empfindungswörter können also kognitive Komponenten haben. Ferner zeigt, wie eben angedeutet, dieses Beispiel eine Querverbindung zwischen einem Satzwort, dem Empfindungswörtchen pfui, und garstig, einem affektiven Adjektiv, an. Garstig ist, so lernt das Kind, wozu man pfui sagt; wie auch vice versa. Solche Querverbindungen, die zwischen scheinbar ganz entlegenen semantisch-lexikalischen ( $t a t s a ̄ c h l i c h$ aber nur grammatisch so verschiedenen) Bereichen existieren, sind allein in Texten zu erkennen; dies ein Hinweis darauf, daß die forschungspraktisch relevante Wortsemantik philologisch sein muß.

14 Wierzbicka (1991, S. 313) deutet die Wahrnehmung des deutschen pfui (wie auch des englischen phew und des skandinavischen $f y$ ) als die einer lautmalerischen Nachahmung "(of) an attempt to breathe out of one's nose a repulsive smell"; demgegen über würden aber polnisch $t$ fu und russisch $t$ 'fu als "imitations of an act of spitting" wahrgenommen.

15 Ewald Lang weist (mdl.) darauf hin, daß die Empfindungswörter oft und zwar in vielen Sprachen phonologisch-phonotaktisch aus der Reihe tanzen. Beispiele dafür gibt Stankiewicz (1964, S. 253), Literatur dazu nennt Mithun (1982, S. 49). Nach Ehlich (1986, S. 211f.) ist ihre besondere phonemische Kombinatorik ein formales Merkmal von Interjektionen überhaupt, und also nicht allein von emotiven; Ehlich (S. 36ff.) deutet außerdem die Intonationsstruktur von manchen der von ihm behandelten Interjektionen als "Ton" (i.S. der "Töne", die in den "Tonsprachen" distinktiv sind) im Deutschen: ein extremes Aus-der-Reihe-Tanzen.

16 Oder für den Sprecher und den Hörer und vielleicht sogar für jeden (normal empfindenden) Menschen. Festzustehen scheint mir, daß ein Adressant mit 
einem Adjektiv wie niedlich ebenso den eigenen Affekt zum Ausdruck bringt wie auch an seine Adressaten appelliert, diesen Affekt zu teilen; ferner aber, $\mathrm{da} \beta$ er, wenn das nicht gelingt und wenn die ausgedrückte affektive Eigenschaft bestritten wird (z.B. mit dem Ausruf Das ist doch nicht niedlich!), er dann stets die Rückzugsposition hat, dafl er sagen kann: Ich finde das aber niedlich. Doch bietet dieser Rückzug keinen absoluten Schutz vor jeglicher Kritik (man kann z.B. insistieren: Wie kannst du das denn niedlich finden?! Das ist doch ...?). Auch die ausgedrūckte Emotion fällt nicht ins Reich der absoluten subjektiven Freiheit.

17 Während der Begriff des kausativen oder faktitiven Verbs seit langem eingeführt ist, scheint es den Begriff des kausativen Adjektivs noch nicht zu geben. Der Gedanke allerdings, daß es so etwas gibt, ist, wenn man danach sucht, zu finden im dritten Band der Deutschen Wortbildung, wo eine Formel mit dem Index kaus erscheint (Kühnhold/Putzer/Wellmann 1978, S. 277) und wo auf einer halben Seite einige der deutschen kausativen Adjektive - in attributiver Verwendung - als kausativ erläutert werden, u.a. (ich zitiere jeweils das Verwendungsbeispiel und in Klammern die Bedeutungsparaphrase): schwindlige Lust ("erregt Schwindel”), abortiver Eingriff („,bewirkt einen Abortus"), ekelhafter Geruch („erregt Ekel"), abscheulicher Gegenstand („erregt Abscheu”), ruinöser Wettbewerb ("bewirkt einen Ruin”), furchtbarer Zorn ("erregt Furcht"), interessanter Fall ("ruft Interesse hervor"), profitables Geschäft ("bringt Profit”). Einige der hier genannten kausativen Adjektive sind sogar affektive kausative Adjektive (ekelhaft, abscheulich, furchtbar, interessant, vielleicht auch schwindlig). Andere Arten kausativer Adjektive sind hier nicht zu finden, weil in diesem Werk zur deutschen Wortbildung natürlich nur die motivierten Adjektive abgehandelt werden. Beispielsweise giftig wäre kausativ zu deuten als: was vergiftet, d.h. was bewirkt, daß man krank wird oder stirbt. - Analog zu kausatives Adjektiv ist der Begriff des kausativen Substantivs zu bilden. Gift z.B. ist durch seine Wirkung definiert (im DUW s.v. durch die Bedeutungsparaphrase: „... Stoft, der ... eine schädliche, zerstörende, tōdliche Wirkung hat ${ }^{p}$ ), also als etwas, was bewirkt, daß diese Wirkung eintritt. Zu kausativen affektiven Substantiven s.u.

18 Weitere Exempel solcher kausativen Adjektive: amüsant (was amüsiert), langweilig (was macht, daB man sich langweilt), tröstlich (was tröstet), sympathisch (was Sympathie hervorruft). Das sind motivierte oder semimotivierte Fälle. Doch genauso wäre etwa auch zu deuten komisch (was belustigt). Für Das ist aber komisch! gab es einmal auch im Deutschen die Paraphrase: Das macht mich lachen (man erinnert sich an Faust, der sagt: „Der Casus macht mich lachen"); im Französischen (Cela me fait rire) und Englischen (It makes me laugh) gibt es sie noch heute. Ferner etwa: frech und unverschämt (hier nur zu betrachten unter dem Aspekt des Ausdrucks von Empörung: frech ist, worüber man sich empört und ärgert; ist dies nicht der Fall, dann war es auch nicht frech); gemütlich (Ausdruck eines Wohlbehagens, das durch das Gemütliche erzeugt wird); blöd (i.S. von worüber man sich ärgert und wie man das Wort verwendet, wenn man sich über etwas ärgert; das DUW (s.v.) gibt das Beispiel „Zu blöd, daß ich das vergesen habe" und nennt in der Tat als Synonym von "ugs." blöd auch ärgerlich; (dazu auch die folgende Anmerkung). - Komplementär und spiegelbildlich zu den affektiven kausativen Adjektiven à la niedlich und abscheulich sind die vielen (oft partizipialen) Adjektive, die den emotiven Zustand explizit benennen, der durch das bewirkt wird, was die affek- 
tiven kausativen Wörter als die Quelle des Affekts beschreiben, beispielsweise erstaunt (komplementär zu erstaunlich), verblüfft (zu verblüffend), enttäuscht (zu enttäuschend), aufgeregt ( $\mathrm{zu}$ aufregend), überrascht (zu überraschend), belustigt (zu belustigend). Diese Adjektive entnehme ich der Aufzählung von Rolf (1993), der hier Gordon (1987, S. 112) zitiert und für das Deutsche adaptiert. Gordon ist es aufgefallen, daß Gefühlszustände oft mit Passiv-Partizipien oder davon abgeleiteten Adjektiven beschrieben werden: „Such adjectives describe [a] state or condition in terms of the particular type of operation or change of state that induces it" (S. 113).

19 Neben traurig, freundlich vgl. etwa auch noch lustig (eine lustige Geschichte ist eine solche, die mich belustigt, d.h. lustig macht), ärgerlich (eine ärgerliche Angelegenheit ist eine solche, die mich ärgert, d.h. ärgerlich macht). Ähnlich ist ein warmer Mantel nicht ein Mantel, der selbst warm ist (wie ein warmer Ofen), sondern einer, der gut warm hält, also sozusagen warm macht.

20 Ausnahme: Üblich ist es, von der propositional attitude, d.h. der bezüglich eines Sachverhalts bzw. einer Proposition ausgedrückten Einstellung (auch Sprechereinstellung) zu reden. Dies kann nach Lang (1983), Motsch/Pasch (1987, S. 36ff.) u.a. auch eine affektive Einstellung sein, die sich in Satzadverbien wie hoffentlich und leider ausdrückt, also nicht bloß eine kognitive (die mit Wörtern wie wahrscheinlich oder möglich ausgedrückt wird). $\mathrm{DaB}$ auch andere Wörter als die Satzadverbien, insbesondere Substantive, Einstellungen zum Ausdruck bringen können, hat schon Keller (1977, S. 23ff.; er sagt Haltungen, das ist die andere Standardübersetzung für englisch attitude) behauptet und erwiesen. Aber sein ansonsten oft zitierter Aufsatz ist in diesem Punkt nicht recht beachtet worden. - Der Begriff der affektiven oder emotiven Wörter (affektiven oder emotiven Adjektive, Substantive, Verben und Adverbien), den ich hier im Text verwendet habe, um hervorzuheben, worauf es bei diesen Wörtern ankommt, ist gebildet in Anlehnung an die englischen Begriffe des affective und emotive meaning. $\mathrm{Zu}$ emotive meaning ist bei Alston $(1967,486 \mathrm{ff}$.) Näheres zu finden. Der Begriff affective meaning wird von Leech (1974, S. 16ff.) verwendet; allerdings erkennt Leech eine genuine Affektivbedeutung nur in (vorsichthalber sagt er aber "chiefly") "interjections" (S. 18). Stevenson (1974, S. 139) erklärt die emotive Bedeutung eines Wortes als dessen Potential, „bestimmte Gefühle, Emotionen oder Einstellungen des Sprechers direkt (quasi-interjektional) auszudrücken [und] zugleich ... [bei Adressaten] hervorzurufen". Das ist, wie ich finde, eine treftende Beschreibung.

21 Dagegen steht (s.v. pauvre) im Petit Rort speziell zu Le pauvre, Mon pauvre, Ma pauvre die Erklärung: „exprime la commisération”. Hier wird also eigens auf den Ausdruck einer Emotion und auf die ausgedrũckte Emotion, das Mitleid, abgehoben. Damit macht das Wörterbuch die Querverbindung sichtbar, die hier zwei anscheinend ganz verschiedene Vokabeln in der Tat verbindet: Mitleid ist, wenn jemand arm sagt.

22 Als besonders glückliche Bedeutungsparaphrase für ein Adverb führe ich aus dem gerade (1993) erschienenen Wörterbuch von Götz/Haensch/Wellmann (1993) an, was dort s.v. endlich steht: „1 nur adv; verwendet, um (nach e-r langen Wartezeit) Erleichterung auszudrūcken: Gott sei Dank, wir sind e. da!; Na e.?'. Der Verlag verwendet diesen Eintrag ganz zu recht in seiner Werbung (man vergleiche hier die anderen deutschen Wörterbücher). 
Im Parasprachlichen und Nonverbalen gibt es hier die allergrößten regionalen und gewif auch soziolektalen Unterschiede. Eine Kollegin sagt mir, daß im Berlinischen das Wörtchen sü $\beta$ als Einwortsatz mit folgender Intonation gesprochen wird: hoch angesetzt, dann langsam und genüßlich bis in eine ziemlich tiefe Lage schleifend; dazu gehören die (auf das gedehnte - üüü) gespitzten Lippen, ein wie zum KuB leicht vorgeschobenes Kinn und halbgeschlossene Augen. Alles dies ist in dem mir selbst bekannten Hamburgischen gànzlich anders.

24 Meinen Titel Kognition, Emotion, Intention verdanke ich bezüglich seines dritten Wortes Gisela Harras, der es ganz natürlich schien, daß dieses dritte Wort zu Kognition und Emotion dazugehört; ich bedanke mich bei ihr auch für die Einladung zu diesem Vortrag. - Inzwischen weiß ich, daß der im folgenden beschriebene Zusammenhang von wollen einerseits und sollen andererseits der germanistischen Modalverbforschung alles andere als unbekannt ist. Cathrine Fabricius-Hansen danke ich für den Hinweis auf Gunnar Bech, der (1949, S. 11) formuliert, sollen bezeichne „einen nicht dem Subjekt innewohnenden Willen ... oder die Forderung (den 'Willen') eines Prinzips ...”. Paul Valentin erwähnt in einem Heidelberger Vortrag, daß der Imperativ-Satz (die Form der Äußerung des Willens in Bezug auf einen anderen par excellence) in indirekter Rede stets per Paraphrase mit dem Verbum sollen abgebildet wird, und zitiert das Grimmsche Wörterbuch, wo es (s.v. sollen, Sp. 1468) heißt: „Die grundbedeutung ... ist die einer verpflichtung oder eines 2 wanges, der auf einem fremden willen beruht", und Brinkmann (1962, S. 368), der formuliert: „Das Verbum sollen.setzt eine Richtung voraus, die der von wollen entgegengesetzt ist. Das grammatische Subjekt handelt nicht aus eigener Richtung, sondern empfängt seine Vollzugsrichtung von einer fremden Instanz". Diese Formulierung hat in der Modalverbtheorie Erfolg gehabt. In Verben in Feldern (darauf weist mich Joachim Ballweg hin) ist angegeben, sollen bedeute eine „Obligation” in einem Kontext, der durch „den Standpunkt irgendeiner Instanz, oft durch den des Sprechers, determiniert" sei (Schumacher 1986, S. 635). Ebenso sagt Weinrich (1993, S. 305f.), sollen bezeichne "das Interesse, das eine andere Instanz vom Subjekt der Handlung verlangt" " - Öhlschläger (1989, S. 171f.) listet frühere Publikationen auf, in denen sollen (tendenziell zumindest) als „Konverse von wollen” so interpretiert wird, daß die beiden Sätze Karl soll heute Klavier spielen und Jemand will, daß Karl heute Klavier spielt als äquivalent aufzufassen sind. - Goethes Verse (aus "Urworte. Orphisch”) werden hier zitiert nach: Goethes Werke. (Hamburger Ausgabe). Band I. 12. Aufl. München 1981. S. 360.

25 Martin Durrell macht (mdl.) darauf aufmerksam, daß für das Englische das hier bezüglich Höflichkeit Gesagte in der Übersetzung nicht gilt. Wo man im Deutschen Soll ich ...? fragt, kann man im Englischen problemlos fragen $D o$ you want me to ...? Da sieht man, daß, was höflich ist, besonders doch vom Usus abhängt.

26 Die Verwandlung einer Wollens-Äußerung in eine Sollens-Äußerung bringt immer eine Agens-Tïgung mit sich, und die Rückverwandlung in die Form der Wollens-ÄuBerung kann daher als Bestandteil einer linguistisch instruierten Kunst des „Zwischen-den-Zeilen-Lesens” i.S. von v. Polenz (1985) angesehen werden. 
27 2. Mose 20. Zitiert nach: Die Bibel. Nach der Übersetzung Martin Luthers. Stuttgart 1985. S. 80.

28 Zitiert nach: Immanuel Kant, Grundlegung zur Metaphysik der Sitten. Hamburg 1957. (Philosophische Bibliothek 41). S. 42. - Daß ein Gesetz, als Manifest und Dokument des kollektiven Sollens, immer seinen Ursprung hat in einem Wollen, wissen die Juristen, die bei der Gesetzesinterpretation nach dem „Willen des Gesetzgebers” fragen.

29 Bislang habe ich die Quelle dieser mir erinnerlichen Verse noch nicht aufgefunden. Stammen sie von Heine?

30 Jean Améry, Über Zwang und Unmöglichkeit, Jude zu sein. In: Jean Améry, Jenseits von Schuld und Sühne. Bewältigungsversuche eines Überwältigten. Neuausgabe Stuttgart 1977, S. 135. „Ich glaube nicht”, schreibt Améry, „daß ich unstatthafterweise Auschwitz und die Endlösung schon ins Jahr 1935 rückprojiziere, wenn ich heute diese Überlegungen anstelle"; zum Beweis dafür zitiert er „Juda verrecke!” und ein Spruchband aus dem Jahre 1935: „Keiner soll hungern, keiner soll frieren, aber die Juden sollen krepieren ...” (S. 134f.).

31 Den Begriff deontische Bedeutung habe ich zuerst in einem lexikographischen Diskussionszusammenhang verwendet (Hermanns 1986) und ihn spãter auf politisch relevante Lexik appliziert (Hermanns 1989). Dabei habe ich mich an dem Sprachgebrauch der Logik orientiert, die von der klassischen Aussagenlogik eine Sollenslogik unterscheidet, nämlich die deontische Logik (kurz Deontik). Innerhalb der Linguistik ist es in der Lehre vom Modalverb üblich, daß man vom deontischen Gebrauch (im Gegensatz zum epistemischen Gebrauch) von sollen, dürfen usw. redet; diese Unterscheidung könnte man zu einer Unterscheidung von deontischer und epistemischer Bedeutung auch bei anderen Wörtern generalisieren, wo sie aber dann Bedeutungskomponenten (statt Bedeutungsvarianten) meinen würde. Auf das Wort (den Terminus) deontisch kommt es aber dabei überhaupt nicht an. Deshalb sage ich - anstelle von deontisch - oft auch präskriptiv und knüpfe damit an die wohlbekannte Unterscheidung zwischen einer deskriptiven vs. präskriptiven Grammatik an. Diese Unterscheidung ist vermutlich auch schon Hare (1952) bekannt gewesen, der (S. 1ff.) den Begriff prescriptive language - in implizitem Gegensatz zu descriptive language - einführt, allerdings nicht in Bezug auf Lexik. In der mit tlerweile langen Diskussion der Sprachfunktionen, die, durch Jakobson (1960) vermitteilt, an Bühler (1934) anschließt, wāhlt Hymes (1962, S. 59) den Terminus directive (function), was im Englischen vortrefflich ist, ins Deutsche aber (als direktive Funktion bzw. Bedeutung) kaum zu übernehmen; deutsch könnte man entsprechend allenfalls von einer orientierenden bzw. Orientierungsfunktion reden. Hymes nennt außerdem auch konativ, pragmatisch, rhetorisch, persuasiv als mögliche Termini für das Gemeinte; das eine oder andere dieser Wörter trifft man auch in deutscher linguistischer Literatur zuweilen an, wenn auch nur auf Āußerungen angewendet, aber davon scheint für meine Zwecke keines gut geeignet (beispielsweise ist rhetorisch viel zu allgemein und konativ nur noch für Latinisten transparent). Müllers (1993, S. 21) Terminus der gerundivischen Bedeutung ist enger als deontische Bedeutung und trifft deshalb das im folgenden speziell Gemeinte (s.u.) besser. Wenn wir ihn benutzen, bleibt der Terminus deontische Bedeutung frei für eine allgemeinere Verwendung. Damit hätten wir dann in deontisch einen Terminus für solche Fälle, wo mit 
einem Wort ein Sollen - oder auch ein Dürfen und Nicht-Dürfen - ausgesagt wird, aber ohne die Bedeutungskomponente Passiv. Beispielsweise ist es, wie ich meine, ein Bestandteil der Bedeutung solcher Wörter wie fleißig, ehrlich, sparsam, freundlich, mutig, klug, daf man so sein bzw. sich verhalten soll, wie diese Wörter sagen; und nicht faul, verlogen, verschwenderisch, unfreundlich, feige, dumm sein oder sich verhalten darf. Das pflegt in der Semantik (wenn es überhaupt gesagt wird) so gesagt zu werden, daB man hier von einer positiven Wertung redet. Aber dieses sozusagen abgeklärte Wort sagt, wie ich finde, völlig unzureichend, daß die Eigenschaften, die die angeführten Adjektive nennen, in der deutschen und der europäischen Kultur gefordert werden, bei Gelegenheit auch eingeklagt bzw. vorgeworfen. Und nicht - in interesseloser Objektivităt gewissermaßen - bloß gemessen und gewichtet und für gut bzw. schlecht befunden, ohne Handlungskonsequenzen.

32 Historisch ist hier zu vermuten, daß die Bildungen auf -wert (wie die auf - würdig usw., s.u.) ihre deutsche Existenz, soweit sie eine Gerundivbedeutung haben, auch einem Übersetzungsnotstand $z u$ verdanken haben: der Verlegenheit, das Gerundiv aus dem Lateinischen ins Deutsche ūbersetzen zu mūssen. So ist lobenswert ein Wort, das das lateinische laudandus (s.o.) (und das gleichbedeutende laudabilis) vollkommen angemessen übersetzt; vermutlich ist es auch zu diesem Zweck geschaffen worden.

\section{Dieses Beispiel gibt (mdI.) Herbert Ernst Wiegand.}

34 Zu den das deontische Bewußtsein prägenden Vokabeln gehört seit etwa 1970 auch Umwelt (Hermanns 1991). Zu Nation und Deutschland vgl. Busse (1993, S. 14): „Der Begriff der Nation bezeichnete daher in Deutschland im 19. Jahrhundert immer noch etwas Zukünftiges, etwas, auf das sich die Hoffnungen richteten und das erst herzustellen sei - es war ein Sollensbegriff, kein Seinsbegriff."

35 Allerdings kann man hier auch erklären: Es zieht! ist als Phraseologismus lexikalisiert und bedeutet daher schon von vornherein Mach bitte schleunigst $z u$, was (Fenster oder Tür) du gerade aufgemacht hast. Auch so hätte Es zieht! eine deontische Bedeutung.

36 Ödön von Horváth, Gesammelte Werke. Band 6. Frankfurt am Main 1972, S. 309.

37 Mit dieser Unterscheidung folge ich dem schon zitierten Aufsatz von Motsch/Pasch (1987), die das faszinierende Verwirrspiel mit den Grice'sche Intentionen paradoxerweise dadurch einer Klārung näher bringen, daß sie eine zusätzliche Intention des Sprechers postulieren: „Wenn ein Sprecher will, daß ein Hörer etwas tut, so muß er auch wollen, daB der Hörer das tun will" (S. 26). Also gibt es nach Motsch/Pasch drei mit einer Äußerung verknüpfte Intentionen, die ein Sprecher $S$ bezüglich eines Hörers $H$ hat: $S$ will, daß $H$ erkennt, d.h. versteht, was er tun soll; $S$ will, daß $H$ akzeptiert, was er tun soll, d.h. $H$ soll dies auch selber wollen; $S$ will, daB $H$ tut, was er tun soll (wenn er etwas tun soll). - Und wenn $H$ dies nun nicht kann und wenn $S$ das auch vielleicht von vornherein schon wuBte? Dann muß es eben dabei bleiben, daß $\mathrm{H}$ sich lediglich den Wunsch von $\mathrm{S}$ zu eigen macht. Oft beschrānkt sich die Funktion von Äußerungen darauf, bestimmte volitive (wie auch kognitive oder affek- 
tive) Haltungen (attitudes) herbeizuführen oder auch nur zu bestärken oder abzuschwächen.

38 Selbst noch in der logischen Deontik (deontischen Logik) bleibt der Kern jedes (Sollens-) Satzes als Proposition frei von jeglicher Sollens-Bedeutung; das Deontische kommt erst (und nur) durch die deontischen Operatoren (analog zu den Modal-Operatoren) in den Satz hinein, wenn sie auf die Propositionen angewendet werden. Ähnlich ist es auch bei Austin, wo ja der locutionary act das ist, was noch bleibt, wenn man von der illokutiven Bedeutung (illocutionary force) einer Äußerung (eines Satzes) abstrahiert, was so gedacht ist, daß die jeweilige illocutionary force zur Bedeutung (meaning) einer Lokution hinzugefügt wird; und nicht etwa schon darin steckt. Ähnlich ist es auch in der Spechhandlungstheorie der Berliner Schule (vgl. etwa Motsch/Pasch 1987). 


\section{Literatur}

Alston, William P. (1967): Art. „Emotion and Feeling” und „Emotive Meaning". In: Edwards, Paul (Hg.): The Encyclopedia of Philosophy. Bd. 1. New York/London. S. 479-493.

Ammann, Hermann (1988): Die drei Sinndimensionen der Sprache. Ein kritisches Referat über die Sprachtheorie Karl Bühlers. In: Eschbach, Achim (Hg.): Karl Būhler's Theory of Language. Amsterdam/Philadelphia. S. 53-76.

Austin, J.L. (1962): How to Do Things with Words. Oxford.

Bech, Gunnar (1949): Das semantische System der deutschen Modalverba. In: Travaux du cercle linguistique de Copenhague 4, S. 3-46.

Bierwisch, Manfred (1980): Semantic Structure and Illocutionary Force. In: Searle, John R./Kiefer, Ferenc/Bierwisch, Manfred (Hg.): Speech Act Theory and Pragmatics. Dordrecht/Boston/London. S. 1-35.

Black, Max (1973): Sprache. Eine Einführung in die Linguistik. München.

Brinkmann, Hennig (1962): Die deutsche Sprache. Gestalt und Leistung. Düsseldorf.

Bühler, Karl (1933): Die Axiomatik der Sprachwissenschaften. Zit. nach der Buchausgabe 2. Aufl. Frankfurt a.M. 1976.

Bühler, Karl (1934): Sprachtheorie. Die Darstellungsfunktion der Sprache. Jena 1934.

Busse, Dietrich (1993): Deutschland, die "schwierige Nation” - Mythos oder Wirklichkeit? In: Reiher, Ruth/Läzer, Rüdiger (Hg.): Wer spricht das wahre Deutsch? Erkundungen zur Sprache im vereinigten Deutschland. Berlin. S. 8-27.

Busse, Winfried (1975): Funktionen und Funktion der Sprache. In: SchliebenLange, Brigitte (Hg.): Sprachtheorie. Hamburg. S. 207-240.

Dieckmann, Walther (1979): K.O. Erdmann und die Gebrauchsweisen des Ausdrucks "Konnotationen" in der linguistischen Literatur. Zitiert nach: Dieckmann, Walther: Politische Sprache - Politische Kommunikation. Vorträge, Aufsätze, Entwürfe. Heidelberg 1981. S. 78-136.

Ehlich, Konrad (1986): Interjektionen. Tübingen. (Linguistische Arbeiten 111).

Erdmann, Karl Otto (1900): Die Bedeutung des Wortes. Aufsätze aus dem Grenzgebiet der Sprachpsychologie und Logik. 4. Aufl. Leipzig 1925. Neudruck Darmstadt 1966.

Fiehler, Reinhard (1990): Kommunikation und Emotion. Theoretische und empirische Untersuchungen zur Rolle von Emotionen in der verbalen Interaktion. Berlin/New York.

Gordon, Robert M. (1987): The Structure of Emotions. Investigations in Cognitive Philosophy. Cambridge.

Hare, R.M. (1952): The Language of Morals. Oxford.

Hermanns, Fritz (1986): Appellfunktion und Wörterbuch. Ein lexikographischer Versuch. In: Wiegand, Herbert Ernst (Hg.): Studien zur neuhochdeutschen Lexikographie VI.1. Hildesheim/Zürich/New York. (Germanistische Linguistik 84-86). S. 151-182.

Hermanns, Fritz (1987): Begriffe partiellen Verstehens. In: Wierlacher, Alois (Hg.): Perspektiven und Verfahren interkultureller Germanistik. München. S. 611-627. 
Hermanns, Fritz (1989): Deontische Tautologien. Ein linguistischer Beitrag zur Interpretation des Godesberger Programms (1959) der Sozialdemokratischen Partei Deutschlands. In: Klein, Josef (Hg.): Politische Semantik. Bedeutungsanalytische und sprachkritische Beiträge zur politischen Sprachverwendung. Opladen. S. 69-149.

Hermanns, Fritz (1991): „Umwelt”. Zur historischen Semantik eines deontischen Wortes. In: Busse, Dietrich (Hg.): Diachrone Semantik und Pragmatik. Untersuchungen zur Erklärung und Beschreibung des Sprachwandels. Tübingen. (Germanistische Linguistik 113). S. 235-257.

Hermanns, Fritz (1992): „Materie” ist nicht „Gegenstand”. Bemerkungen zur deutschen Übersetzung des "Cours de linguistique générale" von Ferdinand de Saussure. In: Anschütz, Susanne R. (Hg.): Texte, Sātze, Wörter und Moneme. Heidelberg. S. 283-289.

Hymes, Dell (1962): Die Ethnographie des Sprechens. Zitiert nach: Hymes, Dell (1979): Soziolinguistik. Zur Ethnographie der Kommunikation. Frankfurt a.M. S. 29-97.

Jäger, Ludwig/Plum, Sabine (1988): Historisches Wörterbuch des deutschen Gefühlswortschatzes. Theoretische und methodische Probleme. In: Jäger, Ludwig (Hg.): Zur historischen Semantik des deutschen Gefühlswortschatzes. Aachen. S. 5-55.

Jãger, Ludwig/Plum, Sabine (1989): Probleme der Beschreibung von Gefühlswörtern im allgemeinen einsprachigen Wörterbuch. In: Hausmann, Franz Josef u.a. (Hg.): Wörterbücher. Dictionaries. Dictionnaires. Ein internationales Handbuch zur Lexikographie. Erster Teilband. Berlin/New York, S. 849-855.

Jakobson, Roman (1960): Linguistics and Poetics. In: Sebeok, Thomas A (Hg.): Style in Language. Cambridge (Mass.). S. 350-377.

Keller, Rudi (1977): Kollokutionäre Akte. In: Germanistische Linguistik 1-2/77, S. 3-50.

Keller, Rudi (1992): Zeichenbedeutung und Bedeutungswandel. In: Zeitschrift für Semiotik 14, S. 327-366.

Kövecses, Zoltán (1990): Emotion Concepts. New York/Berlin/Heidelberg usw.

Kubczak, Hartmut (1984): Bühlers 'Symptomfunktion'. In: Zeitschrift für romanische Philologie 100, S. 1-25.

Kühnhold, Ingeburg/Putzer, Oskar/Wellmann, Hans (1978): Deutsche Wortbildung. Typen und Tendenzen in der Gegenwartssprache. Dritter Hauptteil. Das Adjektiv. Düsseldorf. (Sprache der Gegenwart 43).

Lakoff, George/Johnson, Mark (1980): Metaphors we live by. Chicago/London.

Lang, Ewald (1983): Einstellungsausd rücke und ausgedrückte Einstellungen. In: Ruzicka, Rudolf/Motsch, Wolfgang (Hg.): Untersuchungen zur Semantik. Berlin. (studia grammatica XXII). S. 305-341.

Leech, Geoffrey (1974): Semantics. Harmondsworth.

Liebert, Wolf-Andreas (1992): Metaphernbereiche der deutschen Alltagssprache. Kognitive Linguistik und die Perspektiven einer Kognitiven Lexikographie. Frankfurt a.M. usw.

Ludwig, Klaus-Dieter (1991): Markierungen im allgemeinen einsprachigen Wörterbuch des Deutschen. Ein Beitrag zur Metalexikographie. Tübingen. (Lexicographica. Series maior 38). 
Mithun, Marianne (1982): The Synchronic and Diachronic Behavior of Plops, Squeaks, Croaks, Sighs, and Moans. In: International Journal of American Linguistics 48, S. 49-58.

Morris, Charles (1938): Foundations of the Theory of Signs. Chicago.

Motsch, Wolfgang/Pasch, Renate (1987): Illokutive Handlungen. In: Motsch, Wolfgang (Hg.): Satz, Text, sprachliche Handlung. Berlin. (studia grammatica XXV). S. 11-79.

Müller, Wolfgang (1993): Die Ordnung der Wörter. Kognitive und lexikalische Strukturen. In: Texten \& Schreiben 3/93, S. 20-22.

Öhlschläger, Günther (1989): Zur Syntax und Semantik der Modalverben des Deutschen. Tübingen.

Ortner, Hanspeter (1992): Nachdenken über die Funktionen der Sprache. In: Zeitschrift für germanistische Linguistik 20, S. 271-297.

Polenz, Peter von (1974): Ideolektale und soziolektale Funktionen von Sprache. In: Leuvense Bijdragen 63. S. 97-112.

Polenz, Peter von (1985): Deutsche Satzsemantik. Grundbegriffe des Zwischenden-Zeilen-Lesens. Berlin/New York. (Sammlung Göschen 2226).

Prieto, Luis J. (1975): Pertinence et pratique. Essai de sémiologie. Paris.

Rössler, Gerda (1979): Konnotationen. Untersuchungen zum Problem der Mitund Nebenbedeutung. Wiesbaden.(ZDL Beihefte N.F. 29).

Rolf, Eckard (1993): Emotionen und Handlungen. Erscheint in: Meggle, Georg / Wessels, Ulla (Hg.): Analyomen 1. Vorträge des 1. Kongresses für analytische Philosophie [Arbeitstitel]. Berlin/New York.

Stankiewicz, Edward (1964): Problems of Emotive Language. In: Sebeok, Thomas A. / Hayes, Alfred S. / Bateson, Mary Catherine (Hg.), Approaches to Semiotics. London/The Hague/Paris. S. 239-264.

Stevenson, C. L. (1974): Die emotive Bedeutung ethischer Ausdrücke. In: Grewendorf, Günther / Meggle, Georg (Hg.): Seminar: Sprache und Ethik. Frankfurt a.M. S. 116-139.

Teubert, Wolfgang (1991): Zur Entstehung des Schuldgefühls im 19. Jahrhundert. In: Wimmer, Rainer (Hg.): Das 19. Jahrhundert. Sprachgeschichtliche Wurzeln des heutigen Deutsch. Berlin/New York. (Institut für deutsche Sprache. Jahrbuch 1990). S. 448-471.

Vigener, Gerhard (1978): Die Folgen der Isolierung semiotischer Dimensionen. In: Arbeitsgruppe Semiotik (Hg.): Die Einheit der semiotischen Dimensionen. Tübingen. S. 123-154.

Weinrich, Harald (1993): Textgrammatik der deutschen Sprache. Mannheim/Leipzig/Wien/Zürich.

Wierzbicka, Anna (1991): Cross-Cultural Pragmatics. The Semantics of Human Interaction. Berlin/New York.

Wittgenstein, Ludwig (1921): Logisch-philosophische Abhandlung. Tractatus logico-philosophicus. Zit. (mit Angabe der Satznummer) nach der Ausgabe Frankfurt a.M. 1984. 


\section{Wörterbücher}

Adelung, Johann Christoph (1808): Grammatisch-kritisches Wörterbuch der Hochdeutschen Mundart. ... Erster Theil, von A-E. Wien.

Campe, Joachim Heinrich (1807): Wörterbuch der Deutschen Sprache. Erster Theil. Braunschweig.

DUW $=$ Duden. Deutsches Universalwörterbuch. 2. Auflage. Mannheim usw. 1989.

Grimm, Jacob und Wilhelm (1905): Deutsches Wörterbuch. Zehnten Bandes erste Abtheilung. Leipzig.

Götz, Dieter/Haensch, Günther/Wellmann, Hans (Hg.) (1993): Langenscheidts Grofwörterbuch Deutsch als Fremdsprache. Berlin usw.

Klappenbach, Ruth/Steinitz, Wolfgang (Hg.) (1976): Wörterbuch der deutschen Gegenwartssprache. 5. Band. Berlin.

Muthmann, Gustav (1988): Rückläufiges deutsches Wörterbuch. Handbuch der Wortausgänge im Deutschen, mit Beachtung der Wort- und Lautstruktur. Tübingen.

Paul, Hermann (1992): Deutsches Wörterbuch. 9., vollständig neu bearbeitete Auflage. Tübingen.

Le Petit Robert. Dictionnaire alphabétique \& analogique de la langue française. Paris 1970.

Sanders, Daniel (1860): Wörterbuch der Deutschen Sprache. Erster Band. AK. Leipzig.

Schumacher, Helmut (Hg.) (1986): Verben in Feldern. Valenzwörterbuch zur Syntax und Semantik deutscher Verben. Berlin/New York. (Schriften des Instituts für deutsche Sprache 1). 